\title{
Nonlinear Dynamics of a PI Hydroturbine Governing System with Double Delays
}

\author{
Hongwei Luo, ${ }^{1}$ Jiangang Zhang, ${ }^{2}$ Wenju Du, ${ }^{3}$ Jiarong Lu, ${ }^{4}$ and Xinlei $\mathrm{An}^{2}$ \\ ${ }^{1}$ School of Information Engineering, Gansu Forestry Technological College, Tianshui, Gansu 741020, China \\ ${ }^{2}$ School of Mathematics and Physics, Lanzhou Jiaotong University, Lanzhou, Gansu 730070, China \\ ${ }^{3}$ School of Traffic and Transportation, Lanzhou Jiaotong University, Lanzhou, Gansu 730070, China \\ ${ }^{4}$ School of Applied Mathematics, Xinjiang University of Finance \& Economics, Wulumuqi, Xinjiang 830000, China
}

Correspondence should be addressed to Jiangang Zhang; zhangjg7715776@126.com

Received 11 February 2017; Revised 3 July 2017; Accepted 26 July 2017; Published 12 September 2017

Academic Editor: Yongji Wang

Copyright (c) 2017 Hongwei Luo et al. This is an open access article distributed under the Creative Commons Attribution License, which permits unrestricted use, distribution, and reproduction in any medium, provided the original work is properly cited.

\begin{abstract}
A PI hydroturbine governing system with saturation and double delays is generated in small perturbation. The nonlinear dynamic behavior of the system is investigated. More precisely, at first, we analyze the stability and Hopf bifurcation of the PI hydroturbine governing system with double delays under the four different cases. Corresponding stability theorem and Hopf bifurcation theorem of the system are obtained at equilibrium points. And then the stability of periodic solution and the direction of the Hopf bifurcation are illustrated by using the normal form method and center manifold theorem. We find out that the stability and direction of the Hopf bifurcation are determined by three parameters. The results have great realistic significance to guarantee the power system frequency stability and improve the stability of the hydropower system. At last, some numerical examples are given to verify the correctness of the theoretical results.
\end{abstract}

\section{Introduction}

It is well known that the hydroturbine system is the nonminimum phase system and is operated in a complex condition, constituted by controller and governor. The parameters of the hydroturbine system would change significantly under different operating conditions. According to specific goals, controlled system is researched within a given range. There exist two regulators: PID regulator (Proportion Integration Differentiation regulator) and the soft type feedback regulator (proportional-integral regulator), respectively [1]. Although the characteristics of the PID regulator are simple structure and adaptable and easily adjusting parameters, the regulation law of PID is not efficient. PI regulator is a closed-loop system with phase lag, which can reach steady state by using parameter setting. The PI regulator possesses the properties of the optimal regulator and good robustness. Furthermore, the PI regulator is easy to use in the field, similar to the parameter setting method of the PID regulator. At present, the PI regulator plays a vital role in maintaining the stability of electrical systems and is widely used in China $[2,3]$. On account of lacking systematic management, it is a challenge to maintain the stability of a large hydroelectric station [4-6]. Many efforts are focused on constructing different mathematical models of the hydroturbine governing system and analyzing the stability and the bifurcation phenomena [7-16]; for example, Ling and Tao [13] analyzed the stability and the bifurcation phenomena of a proportional-integral(PI-) (controller) type speed hydroturbine governing system with saturation.

Over the past one decade, many researchers have paid great attention to analyzing dynamic characteristic when the parameters of the hydroturbine systems are changed. For instance, using PI controller, Silva et al. [17] have revealed the problem of stabilizing of a first-order plant with time delay and obtained the stabilizing PI gain values. Shu and Pi [18] introduced a PID neural network (PIDNN) with control time delay and gave examples of analysis. Strah et al. [19] designed a speed and active power controller of hydroturbine units; some controller parameters were obtained. Li and Zhou [20] 
developed a gravitational search algorithm (GSA), which was applied to parameter identification of the hydraulic turbine governing system (HTGS), and analyzed the stability of the power system. Jiang et al. [5] proposed a deterministic chaotic mutation evolutionary programming (DCMEP) method to efficiently optimize the PID parameters of the hydroturbine governing systems. Utilizing a maximum peak resonance specification method, a new PID controller for automatic generation control (AGC) of hydroturbine power systems was presented by Khodabakhshian and Hooshmand [21]. On the basis of necessary and sufficient condition, Liu et al. [22] proposed a new method to analyze the stability of automatic generation control (AGC) systems with commensurate delays. Zhang et al. [23] analyzed a PID-type load frequency control (LFC) scheme by using delay-dependent robust method. Based on state space equations, Chen et al. [24] studied the nonlinear dynamical behaviors of a novel hydroturbine mode with the effect of the surge tank. Xu et al. [25] proposed a Hamiltonian model of the hydroturbine governing system, which included fractional item and time-lag, and explored the effect of the fractional item and the timelag on the dynamic variables of the hydroturbine governing system. Wang et al. [26] studied a novel fractional-order Francis hydroturbine governing system with time delay and verified the effects of the fractional item and time delay on the system by the principle of statistical physics, respectively. The stability and Hopf bifurcation of a Goodwin model with four different delays were investigated by Zhang et al. [27]. Zhang et al. [28] analyzed a hydroturbine governing system in the process of load rejection transient and got the stable regions of the hydroturbine governing system by means of numerical simulations.

However, the existence of a Hopf bifurcation is rarely reported in proportional-integral (PI) type hydroturbine generating system with time delay. In the paper, we generate a PI hydroturbine governing system with saturation and double delays. The nonlinear dynamic behavior of the system is analyzed. The scope of some parameter values is obtained to maintain the stability of the system, which has great realistic significance in a small hydropower station.

The basic structure of the rest paper is as follows. In Section 2, we present a new PI hydroturbine governing system, which is affected by the speed control delay of the generator and the displacement-control delay of the servomotor. In Section 3, the stability of equilibrium points and Hopf bifurcation for PI hydroturbine governing system are investigated in the four different delay cases, respectively. The stability and direction of the Hopf bifurcation are illustrated in Section 4. Numerical simulations are given to support our theory by Matlab software in Section 5. Finally, a brief discussion is given in Section 6.

\section{Model Description}

We study a PI type hydroturbine governing system with saturation and time delay. The structure of the hydroturbine governing system is shown in Figure 1.
The transfer function of soft feedback regulator is

$$
G_{\mathrm{PI}}(s)=K_{P}+\frac{K_{I}}{s} .
$$

We use an approximate linearization approach for the hydroturbine governing system, which is a first-order mathematical model. Moreover the system is set in small perturbation. Therefore, we have

$$
\begin{aligned}
G_{t}(s) & =e_{y} \frac{1-\left(e_{q y} e_{h} / e_{y}-e_{q h}\right) T_{\omega} s}{1+e_{q h} T_{\omega} s}, \\
G_{s} & (s)=\frac{1}{T_{a} s+e_{g}-e_{x}},
\end{aligned}
$$

where $G_{t}(s)$ is used in a nonelastic water column model $[29,30] . G_{s}(s)$ is the transfer function from the hydroturbine moment to the speed of the generator. $e_{q y}$ is servomotor stroke transfer coefficient of the flow rate. $e_{y}$ is servomotor stroke transfer coefficient of the turbine torque. $e_{h}$ is the transfer coefficient of the torque on the water head of a turbine. $e_{q h}$ is the transfer coefficient of the flow rate on the water head of a turbine. $T_{\omega}$ is the water inertia time constant of a pressure guide-water system. $s$ is the strength of the elastic water hammer effect. $T_{a}$ is the sum of the machine starting time and load time constants. $e_{g}$ is the load self-regulation factor. $e_{x}$ is the transfer coefficient of a speed on the turbine torque. $e$ is equal to $e_{q y} e_{h} / e_{y}-e_{q h}$.

If we neglect the load perturbations, then we obtain the state space equations as

$$
\begin{aligned}
\dot{x}_{1}= & x_{2}+-\frac{e_{y} e}{e_{q h} T_{a}} y \\
\dot{x}_{2}= & -\frac{e_{n}}{e_{q h} T_{\omega} T_{a}} x_{1}-\frac{T_{a}+e_{n} e_{q h} T_{\omega}}{e_{q h} T_{\omega} T_{a}} x_{2} \\
& +\left(-\frac{e_{y}}{e_{q h} T_{\omega} T_{a}}+\frac{\left(T_{a}+e_{n} e_{q h} T_{\omega}\right) e_{y} e}{e_{q h}^{2} T_{\omega} T_{a}^{2}}\right) y,
\end{aligned}
$$

where $e_{n}$ is equal to $e_{g}-e_{x}$.

When the nonlinear part can be shown by the nonlinear function $y=N\left(x_{3}-K_{P} x_{1}\right)$, then we have the nonlinear state equation, which is a closed-loop system; we define $x_{3}$ as a state variable,

$$
\dot{x}_{3}=K_{P} \dot{C}+K_{I}\left(C-x_{1}\right),
$$

where $K_{P}$ is the proportional component; $K_{I}$ is the integral component.

Next, when $C=0$, by combining (3) and (4), we have the state space equations of the PI hydroturbine governing system with saturation as

$$
\begin{aligned}
\dot{x}_{1}(t)= & x_{2}(t)+b_{1} N\left(x_{3}(t)-K_{P} x_{1}(t)\right) \\
\dot{x}_{2}(t)= & -a_{0} x_{1}(t)-a_{1} x_{2}(t) \\
& +\left(b_{0}-a_{1} b_{1}\right) N\left(x_{3}(t)-K_{P} x_{1}(t)\right) \\
\dot{x}_{3}(t)= & -K_{I} x_{1}(t),
\end{aligned}
$$




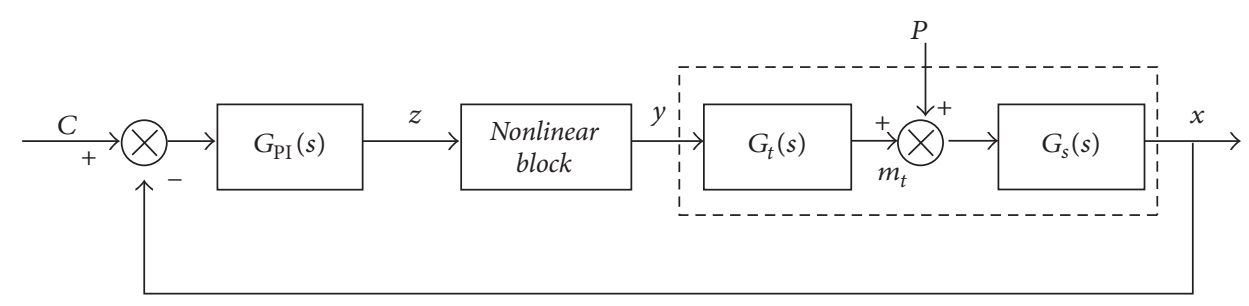

FIGURE 1: Structure of the hydroturbine governing system.

where $a_{1}=\left(T_{a}+e_{n} e_{q h} T_{\omega}\right) / e_{q h} T_{\omega} T_{a}, a_{0}=e_{n} / e_{q h} T_{\omega} T_{a}$, $b_{1}=-e_{y} e / e_{q h} T_{a}, b_{0}=-e_{y} / e_{q h} T_{\omega} T_{a}, e=e_{q y} e_{h} / e_{y}-$ $e_{q h}$, and $N$ is the constant of nonlinear part and equal to 1.

Although Ling and Tao [13] have investigated the existence and direction of the Hopf bifurcation, for the PI hydroturbine governing system, speed control delays and the displacement delays of the servomotor are never considered in the previous studies. In the paper, we consider the dynamics of the system (5) with two different delays. Therefore, we have the following PI hydroturbine governing system as

$$
\begin{aligned}
\dot{x}_{1}(t)= & x_{2}(t)+b_{1} N\left(x_{3}(t)-K_{P} x_{1}\left(t-\tau_{1}\right)\right) \\
\dot{x}_{2}(t)= & -a_{0} x_{1}(t)-a_{1} x_{2}(t) \\
& +\left(b_{0}-a_{1} b_{1}\right) N\left(x_{3}\left(t-\tau_{2}\right)-K_{P} x_{1}(t)\right) \\
\dot{x}_{3}(t)= & -K_{I} x_{1}(t),
\end{aligned}
$$

where $\tau_{1}$ is the speed control delay of the generator. $\tau_{2}$ is the displacement-control delay of the servomotor.

\section{Stability Analysis and Hopf Bifurcation}

Usually, it is not easy to find out its accurate solutions. It is sufficient to research the stability of $E^{*}=\left(x_{1}^{*}, x_{2}^{*}, x_{3}^{*}\right)$; we only consider $E_{0}=(0,0,0)$. At equilibrium point $E_{0}$, the Jacobi matrix of the system (6) is

$$
\left[\begin{array}{ccc}
-b_{1} N \cdot K_{P} \cdot e^{-\lambda \tau_{1}} & 1 & b_{1} N \\
\left(a_{1} b_{1}-b_{0}\right) N \cdot K_{P}-a_{0} & -a_{1} & \left(b_{0}-a_{1} b_{1}\right) N \cdot e^{-\lambda \tau_{2}} \\
-K_{I} & 0 & 0
\end{array}\right]
$$

Then the associated characteristic equation is

$$
\begin{aligned}
\lambda^{3}+ & A_{1} \lambda^{2}+A_{2} \lambda+A_{3}+\left(A_{4} \lambda^{2}+A_{5} \lambda\right) e^{-\lambda \tau_{1}} \\
& +A_{6} e^{-\lambda \tau_{2}}=0
\end{aligned}
$$

where

$$
\begin{aligned}
& A_{1}=a_{1}, \\
& A_{2}=N K_{P} b_{0}+N K_{I} b_{1}+a_{0}-N K_{P} a_{1} b_{1} \\
& A_{3}=N K_{I} a_{1} b_{1}, \\
& A_{4}=N K_{P} b_{1}, \\
& A_{5}=N K_{P} a_{1} b_{1}, \\
& A_{6}=-N K_{I} a_{1} b_{1}+N K_{I} b_{0} .
\end{aligned}
$$

Next, we investigate the distribution of the roots of (8) with different delay values for $\tau_{1}$ and $\tau_{2}$.

It is apparent that (8) assumes the following form when $\tau_{1}=0, \tau_{2}=0$.

$$
\lambda^{3}+\lambda^{2}\left(A_{1}+A_{4}\right)+\lambda\left(A_{2}+A_{5}\right)+A_{3}+A_{6}=0 .
$$

Furthermore, we propose the Routh-Hurwitz stability criterion; a corresponding certificate shall be found [31].

Lemma 1 (see [32]). The polynomial $L(\lambda)=\lambda^{3}+p_{1} \lambda^{2}+$ $p_{2} \lambda+p_{3}$ with real coefficients has all roots with negative real parts if and only if the numbers $p_{1}, p_{2}, p_{3}$ are positive and the inequality $p_{1} p_{2}>p_{3}$ is satisfied.

According to Lemma 1, all roots of (10) have negative real parts if and only if

(H1) $A_{1}+A_{4}>0, A_{2}+A_{5}>0, A_{3}+A_{6}>0$ and $\left(A_{1}+\right.$ $\left.A_{4}\right)\left(A_{2}+A_{5}\right)>A_{3}+A_{6}$.

So $E^{*}=\left(x_{1}^{*}, x_{2}^{*}, x_{3}^{*}\right)$ is locally asymptotically stable when (H1) holds.

Case $1\left(\tau=\tau_{1}=\tau_{2} \neq 0\right)$. We rewrite (8) as follows:

$$
\begin{aligned}
\lambda^{3} & +A_{1} \lambda^{2}+A_{2} \lambda+A_{3}+\left(A_{4} \lambda^{2}+A_{5} \lambda+A_{6}\right) e^{-\lambda \tau} \\
& =0 .
\end{aligned}
$$

If $\lambda=i \omega$ is a root of (11), then we have

$$
\omega^{10}+\omega^{8} C_{1}+\omega^{6} C_{2}+\omega^{4} C_{3}+\omega^{2} C_{4}+C_{5}=0,
$$


where

$$
\begin{aligned}
& C_{1}=\frac{\left(2 B_{1} B_{2}+B_{6}^{2}-B_{1}^{4}\right)}{B_{1}^{2}}, \\
& C_{2}=\frac{\left(2 B_{1} B_{3}-2 B_{1}^{2} B_{4}+B_{2}^{2}+2 B_{6} B_{7}\right)}{B_{1}^{2}}, \\
& C_{4}=\frac{\left(B_{3}^{2}-2 B_{4} B_{5}+2 B_{7} B_{8}\right)}{B_{1}^{2}}, \\
& C_{3}=\frac{\left(2 B_{2} B_{3}-2 B_{1}^{2} B_{5}-B_{4}^{2}+2 B_{6} B_{8}+B_{7}^{2}\right)}{B_{1}^{2}}, \\
& C_{5}=\frac{\left(-B_{5}^{2}+B_{8}^{2}\right)}{B_{1}^{2}} .
\end{aligned}
$$

Let $z=\omega^{2}$, we define

$$
h(z)=z^{5}+z^{4} C_{1}+z^{3} C_{2}+z^{2} C_{3}+z C_{4}+C_{5} .
$$

From the definition of $h(z)$, we can obtain $h(+\infty)=+\infty$; thus we can get that (14) has at least one positive root if $h(0)=$ $C_{5}<0$ holds.

We assume that (14) has five positive roots, which are defined by $z_{1}, z_{2}, z_{3}, z_{4}$, and $z_{5}$. Then, (12) has five positive roots $\omega_{k}=\sqrt{z_{k}}, \quad k=1,2,3,4,5$.
If we denote

$$
\begin{array}{r}
\tau_{k}^{(i)}=\frac{1}{\omega_{k}}\left\{\arccos \left(\frac{\omega^{4} B_{6}+\omega^{2} B_{7}+B_{8}}{\omega^{4} B_{1}^{2}+\omega^{2} B_{4}+B_{5}}\right)+2 i \pi\right\}, \\
i=0,1,2, \ldots, k=1,2,3,4,5,
\end{array}
$$

then $\pm i \omega_{k}$ is a pair of purely imaginary roots of (8) with $\tau=$ $\tau_{k}^{(i)}$.

Lemma 2. Define $\tau_{0}=\tau_{k}^{(0)}=\min _{k \in\{1,2,3\}}\left\{\tau_{k}^{(0)}\right\}$ and $\omega_{0}=\omega_{k_{0}}$. Let $\lambda(\tau)=\xi(\tau)+i \omega(\tau)$ be the root of $(8)$ near $\tau=\tau_{0}$, satisfying $\xi\left(\tau_{0}\right)=0, \omega\left(\tau_{0}\right)=\omega_{0}$; the following transversality condition holds: $(d \operatorname{Re} \lambda(\tau) / d \tau)_{\tau=\tau_{k}^{(i)}} \neq 0$.

By applying Lemma 2 to (11), we can obtain the following theorem.

Theorem 3. Suppose $H_{2}=P_{R} Q_{R}+P_{1} Q_{1} \neq 0$ holds; with the increasement of delay variable $\tau$ from zero, there is a value of $\tau_{0}$, such that the positive equilibrium point $E^{*}$ is locally asymptotically stable for $\tau \in\left[0, \tau_{0}\right)$ and unstable for $\tau>\tau_{0}$. Moreover, system (6) occurs with a Hopf bifurcation at $E^{*}$ when $\tau=\tau_{0}$.

Case $2\left(\tau_{1} \neq 0, \tau_{2}=0\right)$. We rewrite (8) as follows:

$$
\begin{aligned}
\lambda^{3} & +A_{1} \lambda^{2}+A_{2} \lambda+A_{3}+A_{6}+\left(A_{4} \lambda^{2}+A_{5} \lambda\right) e^{-\lambda \tau_{1}} \\
& =0 .
\end{aligned}
$$

Let $\lambda=i \omega$ be a root of (16), then we have

$$
z^{4}+z^{3} D_{1}+z^{2} D_{2}+z D_{3}+D_{4}=0
$$

where

$$
\begin{aligned}
& D_{1}=\frac{\left(A_{1}^{2} A_{4}^{2}+A_{5}^{2}-A_{4}^{4}-2 A_{2} A_{4}^{2}\right)}{A_{4}^{2}}, \\
& D_{2}=\frac{\left(-2 A_{4}^{2} A_{5}^{2}+A_{1}^{2} A_{5}^{2}+A_{2}^{2} A_{4}^{2}-2 A_{2} A_{5}^{2}-2 A_{1} A_{3} A_{4}^{2}-2 A_{1} A_{4}^{2} A_{6}\right)}{A_{4}^{2}}, \\
& D_{3}=\frac{\left(-A_{5}^{4}+A_{3}^{2} A_{4}^{2}+A_{4}^{2} A_{6}^{2}+A_{2}^{2} A_{5}^{2}+2 A_{3} A_{4}^{2} A_{6}-2 A_{1} A_{3} A_{5}^{2}-2 A_{1} A_{5}^{2} A_{6}\right)}{A_{4}^{2}}, \\
& D_{4}=\frac{\left(A_{3} A_{5}+A_{5} A_{6}\right)^{2}}{A_{4}^{2}} .
\end{aligned}
$$

If we denote

$$
\tau_{k}^{(i)}=\frac{1}{\omega_{k}}\left\{\arccos \left(\frac{\omega^{3}\left(A_{5}-A_{1} A_{4}\right)+\omega\left(A_{3} A_{4}-A_{2} A_{5}+A_{4} A_{6}\right)}{\omega^{3} A_{4}^{2}+\omega A_{5}^{2}}\right)+2 i \pi\right\}, \quad i=0,1,2, \ldots, k=1,2,3,4
$$


then $\pm i \omega_{k}$ is a pair of purely imaginary roots of (8) with $\tau_{1}=$ $\tau_{k}^{(i)}$.

Lemma 4. Define $\tau_{10}=\tau_{k}^{(0)}=\min _{k \in\{1,2,3\}}\left\{\tau_{k}^{(0)}\right\}$ and $\omega_{0}=$ $\omega_{k_{0}}$. Let $\lambda(\tau)=\xi(\tau)+i \omega(\tau)$ be the root of $(16)$ near $\tau_{1}=\tau_{10}$, satisfying $\xi\left(\tau_{10}\right)=0, \omega\left(\tau_{10}\right)=\omega_{0}, z_{k}=\omega_{k}^{2}$ and $h^{\prime}\left(z_{k}\right) \neq 0$; then $\left(d \operatorname{Re} \lambda\left(\tau_{10}\right) / d \tau_{1}\right)_{\tau_{1}=\tau_{k}^{(i)}} \neq 0,\left(d \operatorname{Re} \lambda\left(\tau_{10}\right) / d \tau_{1}\right)$, and $h^{\prime}\left(z_{k}\right)$ have the same sign.

Next, we have to look for the conditions required for (17) to have at least one positive root.

We denote

$$
h(z)=z^{4}+z^{3} D_{1}+z^{2} D_{2}+z D_{3}+D_{4} .
$$

By applying Lemma 1 to (17), we obtain the following theorem.

\section{Theorem 5. For (20), the following result holds.}

(i) If $D_{4}>0$ and $\Delta=D_{1} D_{2} D_{3}-D_{4} D_{1}^{2}-D_{3}^{2} \leq 0$, then the zero solution of system (6) is asymptotically stable for $\tau_{1}>0$.

(ii) If $D_{4}<0$ and $\Delta=D_{1} D_{2} D_{3}-D_{4} D_{1}^{2}-D_{3}^{2}>0$, then $E^{*}=\left(x_{1}^{*}, x_{2}^{*}, x_{3}^{*}\right)$ of system (6) is asymptotically stable for $\tau_{1} \in\left[0, \tau_{10}\right)$, and it is unstable when $\tau>\tau_{10}$.

(iii) If all the conditions stated in (ii) and $h^{\prime}(z) \neq 0$ are satisfied, system (6) occurs with a Hopf bifurcation at $E^{*}=\left(x_{1}^{*}, x_{2}^{*}, x_{3}^{*}\right)$ when $\tau_{1}=\tau_{k}^{(i)}(i=0,1,2, \ldots)$.

Case $3\left(\tau_{1}=0, \tau_{2} \neq 0\right)$. We rewrite (8) as follows:

$$
\lambda^{3}+A_{1} \lambda^{2}+A_{2} \lambda+A_{3}+A_{6} e^{-\lambda \tau_{2}}=0 .
$$

By letting $\lambda=i \omega$ be the root of (21), we have

$$
z^{3}+E_{1} z^{2}+E_{2} z+E_{3}=0
$$

The roots of (22) are considered by Lemma 1 . Without losing generality, we suppose that (22) has three positive roots, which are defined by $z_{1}, z_{2}$, and $z_{3}$.

If we denote

$$
\begin{aligned}
\tau_{k}^{(i)}=\frac{1}{\omega_{k}}\left\{\arccos \left(\frac{A_{1} \omega^{2}-A_{3}}{A_{6}}\right)+2 i \pi\right\}, & \\
& i=0,1,2, \ldots, k=1,2,3,
\end{aligned}
$$

then $\pm i \omega_{k}$ is a pair of purely imaginary roots of (8) with $\tau_{2}=$ $\tau_{k}^{(i)}$.

Define $\tau_{20}=\tau_{k}^{(0)}=\min _{k \in\{1,2,3\}}\left\{\tau_{k}^{(0)}\right\}$ and $\omega_{0}=\omega_{k_{0}}$. Let $\lambda(\tau)=\xi(\tau)+i \omega(\tau)$ be the root of (21) near $\tau_{2}=\tau_{20}$, satisfying $\xi\left(\tau_{10}\right)=0$ and $\omega\left(\tau_{10}\right)=\omega_{0}$, then the following transversality condition holds.

Lemma 6. Suppose that $z_{k}=\omega_{k}^{2}$ and $h^{\prime}\left(z_{k}\right) \neq 0$, then $\left(d \operatorname{Re} \lambda\left(\tau_{20}\right) / d \tau_{2}\right)_{\tau_{1}=\tau_{k}^{(i)}} \neq 0,\left(d \operatorname{Re} \lambda\left(\tau_{20}\right) / d \tau 2\right)_{\tau_{1}=\tau_{k}^{(i)}}$, and $h^{\prime}\left(z_{k}\right)$ have the same sign.
The proof is similar to that of Lemma 4, so we ignore the proofs. By applying Lemma 6 to (21), we have the following theorem.

Theorem 7. As $\tau_{2}$ increases from zero, there exists a critical value $\tau_{20}$, such that $E^{*}$ is locally asymptotically stable for $\tau_{1} \in$ $\left[0, \tau_{20}\right)$ and unstable when $\tau_{2}>\tau_{20}$. Moreover, system (6) occurs a Hopf bifurcation at $E^{*}$ when $\tau_{2}=\tau_{20}$.

Case $4\left(\tau_{1} \neq \tau_{2}, \tau_{1}>0\right.$, and $\left.\tau_{2}>0\right)$. We consider (8) with $\tau_{1}$ in its stability range. Regarding $\tau_{2}$ as a parameter, without losing generality, we consider system (6) in Case 2. Let $\omega i(\omega>0)$ be a root of (8); then we obtain

$$
F_{1}(\omega)+F_{2}(\omega) \sin \left(\omega \tau_{1}\right)+F_{3} \cos \left(\omega \tau_{1}\right)=0
$$

where

$$
\begin{aligned}
F_{1}(\omega)= & \omega^{6}+\omega^{4}\left(A_{1}^{2}+A_{4}^{2}-2 A_{2}\right) \\
& +\omega^{2}\left(A_{2}^{2}+A_{5}^{2}-2 A_{1} A_{3}\right)+A_{3}^{2}-A_{6}^{2}, \\
F_{2}(\omega)= & -2 \omega^{5} A_{4}+\omega^{3}\left(2 A_{2} A_{4}-2 A_{1} A_{5}\right) \\
& +2 \omega A_{3} A_{5}, \\
F_{3}(\omega)= & -2 \omega^{5} A_{4}+2 \omega^{4} A_{1} A_{4}+2 \omega^{3} A_{2} A_{4} \\
& -2 \omega^{2} A_{3} A_{4} .
\end{aligned}
$$

Therefore, utilizing the general Hopf bifurcation theorem for functional differential equations (FDEs) as given in Hale [33], we obtain the following results for system (6).

Theorem 8. Suppose that (24) has at least finite positive roots, $P_{R} Q_{R}+P_{I} Q_{I} \neq 0$ and $\tau_{1} \in\left[0, \tau_{10}\right)$, then the positive equilibrium point $E^{*}$ of system (6) is locally asymptotically stable for $\tau_{2} \in\left[0, \tau_{2 *}\right)$. For $E^{*}$, system (6) undergoes a Hopf bifurcation when $\tau_{2}=\tau_{2 *}$. System (6) has a branch of periodic solutions bifurcating from the zero solution near $\tau_{2}=\tau_{2 *}$.

\section{Stability and Direction of the Hopf Bifurcation}

In the above section, we have studied that PI hydroturbine governing model (6) with double delays undergoes a Hopf bifurcation for $\tau=\tau_{k}^{(i)}(i=0,1,2, \ldots, k=1,2, \ldots)$. In this section, we assume that system (6) undergoes a Hopf bifurcation at $\tau=\tau_{0}^{(i)}(i=0,1,2, \ldots)$. Utilizing the normal form theory and the center manifold reduction, the stability, the direction, and the bifurcation of the periodic solutions are determined.

For convenience sake, we suppose that $\tau_{0}$ is less than $\tau_{2 *}$, where $\tau_{2 *} \in\left[0, \tau_{10}\right)$. Let $u(t)=\left(x_{1}(t), x_{2}(t), x_{3}(t)\right)^{T} \in$ $R^{3}, \quad \tau_{1}=\tau_{0}+\mu$, in which $\mu \in R$, then we transform system (6) into functional differential equations (FDEs) in $C=C\left([-1,0], R^{3}\right)$ as

$$
\dot{x}(t)=L_{\mu}\left(x_{t}\right)+f\left(\mu, x_{t}\right),
$$


where $L_{\mu}: C \rightarrow R^{3}, f: R \times C \rightarrow R^{3}$ are given, respectively. We obtain

$$
\begin{gathered}
L \mu\left(x_{t}\right)=\left(\tau_{0}+\mu\right)\left[\begin{array}{lll}
0 & 1 & c_{1} \\
c_{2} & c_{3} & 0 \\
c_{4} & 0 & 0
\end{array}\right]\left[\begin{array}{l}
\phi_{1}(0) \\
\phi_{2}(0) \\
\phi_{3}(0)
\end{array}\right] \\
+\left(\tau_{0}+\mu\right)\left[\begin{array}{lll}
c_{5} & 0 & 0 \\
0 & 0 & c_{6} \\
0 & 0 & 0
\end{array}\right]\left[\begin{array}{l}
\phi_{1}(-1) \\
\phi_{2}(-1) \\
\phi_{3}(-1)
\end{array}\right], \\
f(u, \phi)=\left(\tau_{0}+\mu\right)\left[\begin{array}{c}
c_{5} \phi_{1}(-1) \\
c_{6} \phi_{3}(-1) \\
0
\end{array}\right],
\end{gathered}
$$

where $c_{1}=b_{1} N, c_{2}=a_{1} b_{1} N K_{P}-b_{0} N K_{P}-a_{0}, c_{3}=-a_{1}, c_{4}=$ $-K_{I}, \quad c_{6}=\left(b_{0}-a_{1} b_{1}\right) N, c_{5}=-b_{1} N K_{P}$ and $\phi=\left(\phi_{1}, \phi_{2}, \phi_{3}\right)^{T} \in$ $C\left([-1,0], R^{3}\right)$.

Utilizing the Riesz representation theorem, there is a $3 \times 3$ matrix-valued function $\eta(\theta, \mu)$ of bounded variation for $\theta \in$ $[-1,0]$, such that

$$
L_{\mu} \phi=\int_{-1}^{0} d \eta(\theta, 0) \phi(\theta),
$$

Indeed, we may take

$$
\begin{aligned}
\eta(\theta, \mu)=\left(\tau_{0}+\mu\right)\left[\begin{array}{lll}
0 & 1 & c_{1} \\
c_{2} & c_{3} & 0 \\
c_{4} & 0 & 0
\end{array}\right] \delta(\theta) \\
-\left(\tau_{0}+\mu\right)\left[\begin{array}{lll}
c_{5} & 0 & 0 \\
0 & 0 & c_{6} \\
0 & 0 & 0
\end{array}\right] \delta(\theta+1),
\end{aligned}
$$

where $\delta$ is the Dirac delta function.

For $\phi \in C^{-1}\left([-1,0], R^{3}\right)$, we define

$$
A^{*} \psi(s)= \begin{cases}-\frac{d \psi(s)}{d s}, & s \in(0,1] \\ \int_{-1}^{0} d \eta^{T}(t, 0) \psi(-t), & s=0\end{cases}
$$

and a bilinear inner product:

$$
\begin{aligned}
\langle\psi(s), \phi(\theta)\rangle= & \bar{\psi}(0) \phi(0) \\
& -\int_{-1}^{0} \int_{\xi=0}^{\theta} \bar{\psi}(\xi-\theta) d \eta(\theta) \phi(\xi) d \xi,
\end{aligned}
$$

where $\eta(\theta)=\eta(\theta, 0) ; A(0)$ and $A^{*}$ are adjoint operators. From the above analysis, we obtain that $q(\theta)$ and $q^{*}(s)$ are eigenvectors of $A$ and $A^{*}$ corresponding to $i \omega_{0} \tau_{k}$ and $-i \omega_{0} \tau_{k}$, respectively. Suppose that $q(\theta)=\left(1, v_{1}, v_{2}\right)^{T} e^{i \omega_{0} \tau_{k} \theta}$ is the eigenvector of $A(0)$ corresponding to $i \omega_{0} \tau_{k}$; then $A(0) q(\theta)=$ $i \omega_{0} \tau_{0} q(\theta)$. It follows from the definition of $A(0), L_{\mu} \phi$, and $\eta(\theta, \mu)$ that

$$
q(\theta)=\left(1, v_{1}, v_{2}\right)^{T} e^{i \omega_{0} \tau_{k} \theta}=q(0) e^{i \omega_{0} \tau_{k} \theta},
$$

and similarly, by definition of $A^{*}$,

$$
q^{*}(\theta)=D\left(1, v_{1}^{*}, v_{2}^{*}\right)^{T} e^{i \omega_{0} \tau_{k} \theta}=q^{*}(0) e^{i \omega_{0} \tau_{k} \theta}
$$

Through a simple calculation, we can obtain

$$
\begin{aligned}
& v_{1}=\frac{-\omega_{0} c_{5} e^{-i \omega_{0} \tau_{k}}+\left(\omega_{0}^{2}+c_{1} c_{4}\right) i}{\omega_{0}}, \\
& v_{2}=\frac{c_{3} c_{5} e^{-\omega_{0} i \tau_{k}}-c_{2}-\omega_{0}^{2}-\left(\omega_{0} c_{3}+c_{5} e^{-\omega_{0} i \tau_{k}}\right) i}{c_{6} e^{-\omega_{0} i \tau_{k}}-c_{1} c_{3}+c_{1} \omega_{0} i}, \\
& v_{1}^{*}=\frac{c_{1} c_{4}+\omega_{0}^{2}-\omega_{0} c_{5} e^{-\omega_{0} i \tau_{k}} i}{c_{2} \omega_{0} i-c_{4} c_{6} e^{-\omega_{0} i \tau_{k}}}, \\
& v_{2}^{*}=\frac{c_{2}+\omega_{0}^{2}-c_{3} c_{5} e^{-\omega_{0} i \tau_{k}}-\left(\omega_{0} c_{3}+c_{5} \omega_{0} e^{-\omega_{0} i \tau_{k}}\right) i}{\left(\omega_{0} i+c_{3}\right) c_{4}} .
\end{aligned}
$$

From (31), one has

$$
\begin{aligned}
& \left\langle q^{*}(s), q(\theta)\right\rangle=\bar{q}^{*}(0) q(0) \\
& \quad-\int_{-1}^{0} \int_{\xi=0}^{\theta} \bar{q}^{*}(\xi-\theta) d \eta(\theta) q(\xi) d \xi \\
& \quad=\bar{D}\left(1, \bar{v}_{1}^{*}, \bar{v}_{2}^{*}\right)\left(1, v_{1}, v_{2}\right)^{T}-\int_{-1}^{0} \int_{\xi=0}^{\theta} \bar{D}\left(1, \bar{v}_{1}^{*}, \bar{v}_{2}^{*}\right) \\
& \cdot e^{-\omega_{0} i \tau_{k}(\xi-\theta)} d \eta(\theta)\left(1, v_{1}, v_{2}\right)^{T} e^{\omega_{0} i \tau_{k} \xi} d \xi=\bar{D}\{1 \\
& \left.+\bar{v}_{1}^{*} v_{1}+\bar{v}_{2}^{*} v_{2}+\tau_{k}\left(c_{5}+c_{6} v_{2}^{*}\right) e^{-\omega_{0} \tau_{k} i}\right\} .
\end{aligned}
$$

Thus we can choose $\bar{D}$ as

$$
\bar{D}=\frac{1}{1+\bar{v}_{1}^{*} v_{1}+\bar{v}_{2}^{*} v_{2}+\tau_{k}\left(c_{5}+c_{6} v_{2}^{*}\right) e^{-\omega_{0} \tau_{k}} i},
$$

such that $\left\langle q^{*}(s), q(\theta)\right\rangle=1$ and $\left\langle q^{*}(s), \bar{q}(\theta)\right\rangle=0$.

Next, through the use of the same notations in Hassard et al. [34], we can calculate the coordinates describing the center manifold $C_{0}$ at $\mu=0$. Therefore, we have the following:

$$
\begin{aligned}
g_{20} & =2 \bar{D} \tau_{k}\left[\left(c_{5}+c_{6} v_{2}^{*}\right) e^{-i \omega_{0} \tau_{k}}+1+c_{3}\right], \\
g_{11} & =2 \bar{D} \tau_{k}\left[\left(c_{5}+c_{6} \bar{v}_{2}^{*}\left|v_{2}\right|^{2}\right) \operatorname{Re}\left\{e^{i \omega_{0} \tau_{k}}\right\}+1+c_{1}\right] \\
g_{02} & =2 \bar{D} \tau_{k}\left[\left(c_{5}+c_{6} v_{2}^{*}\right) e^{i \omega_{0} \tau_{k}}+1+c_{3}\right], \\
g_{21} & =\bar{D} \tau_{k}\left[c_{5}\left(W_{20}^{(1)}(-1)+W_{11}^{(1)}(-1)\right)\right. \\
& \left.+c_{6}\left(W_{20}^{(3)}(-1)+W_{11}^{(3)}(-1)\right)\right]
\end{aligned}
$$


where

$$
\begin{aligned}
& W_{20}(\theta) \\
& =\frac{i g_{20}}{\omega_{0} \tau_{k}} q(0) e^{i \omega_{0} \tau_{k} \theta}+\frac{i \bar{g}_{02}}{3 \omega_{0} \tau_{k}} \bar{q}(0) e^{-i \omega_{0} \tau_{k} \theta} \\
& +E_{1} e^{2 i \omega_{0} \tau_{k} \theta} \\
& W_{11}(\theta) \\
& =-\frac{i g_{11}}{\omega_{0} \tau_{k}} q(0) e^{i \omega_{0} \tau_{k} \theta}+\frac{i \bar{g}_{11}}{\omega_{0} \tau_{k}} \bar{q}(0) e^{-i \omega_{0} \tau_{k} \theta}+E_{2}, \\
& E_{1} \\
& =2\left[\begin{array}{ccc}
c_{5} e^{-2 i \omega_{0} \tau_{k}} & 1 & c_{1}+2 i \omega_{0} \\
c_{2}+2 i \omega_{0} & c_{3}+2 i \omega_{0} & c_{6} e^{-2 i \omega_{0} \tau_{k}} \\
c_{4}+2 i \omega_{0} & 0 & 0
\end{array}\right]^{-1}\left[\begin{array}{c}
c_{5} e^{-i \omega_{0} \tau_{k}} \\
c_{6} e^{-i \omega_{0} \tau_{k}} \\
0
\end{array}\right], \\
& E_{2}=2\left[\begin{array}{ccc}
c_{5} & 1 & c_{1} \\
c_{2} & c_{3} & c_{6} \\
c_{4} & 0 & 0
\end{array}\right]^{-1}\left[\begin{array}{c}
c_{5} \operatorname{Re}\left\{e^{-i \omega_{0} \tau_{k}}\right\} \\
c_{6} \operatorname{Re}\left\{e^{-i \omega_{0} \tau_{k}}\right\} \\
0
\end{array}\right] \text {. }
\end{aligned}
$$

Thus, we get the following quantities:

$$
\begin{aligned}
c_{1}(0) & =\frac{i}{2 \omega_{0} \tau_{k}}\left(g_{20} g_{11}-2\left|g_{11}\right|^{2}-\frac{1}{3}\left|g_{02}\right|^{2}\right)+\frac{1}{2} g_{21}, \\
\mu_{2} & =-\frac{\operatorname{Re}\left\{c_{1}(0)\right\}}{\operatorname{Re}\left\{\lambda^{\prime}(0)\right\}}, \\
T_{2} & =-\frac{\operatorname{Im}\left\{c_{1}(0)\right\}+\mu_{2} \operatorname{Im}\left\{\lambda^{\prime}(0)\right\}}{\omega_{0} \tau_{k}}, \\
\beta_{2} & =2 \operatorname{Re}\left\{c_{1}(0)\right\} .
\end{aligned}
$$

From the above analysis, we have the theorem as follows.

Theorem 9. When $\tau_{k}$ is equal to $\tau_{0}^{i}$, the stability and direction of the Hopf bifurcation for system (6) are confirmed by the parameters $\mu_{2}, T_{2}$, and $\beta_{2}$.

(1) $\mu_{2}$ determines the direction of the Hopf bifurcation: if $\mu_{2}>0$ and $\tau_{k}>\tau_{0}^{0}$, then the Hopf bifurcation is supercritical; if $\mu_{2}>0$ and $\tau_{k}>\tau_{0}^{0}$, then he Hopf bifurcation is subcritical. In both cases, the bifurcating periodic solutions of system (6) exist.

(2) $T_{2}$ determines the period of the bifurcating periodic solutions: if $\mathrm{T}_{2}>0$, the period increases; else the period decreases.

(3) $\beta_{2}$ determines the stability of the bifurcating periodic solutions: the bifurcating periodic solutions are stable (unstable) for $\beta_{2}<0\left(\beta_{2}>0\right)$.

\section{Numerical Example}

Suppose the hydroturbine governing system which is set works under certain operating conditions, the parameters are as follows: $N=1, a_{0}=0.667, a_{1}=2.522, b_{0}=0.311, b_{1}=$ -0.342 . Utilizing the function dde23(), numerical calculations have been performed as follows.

In Case 1, system (6) has two same time delays $\tau$, initial value $x_{0}=(0.05,0.05,0.05), K_{P}=4.5$, and $K_{I}=-1.326$; we can obtain the Hopf bifurcation value $\tau_{0}=0.35 \mathrm{~s}$. When $\tau<\tau_{0}=0.35 \mathrm{~s}$, at the equilibrium $E^{*}=\left(x_{1}^{*}, x_{2}^{*}, x_{3}^{*}\right)$, system (6) is asymptotically stable, and it is unstable when $\tau>\tau_{0}$ are shown in Figures 2(b) and 2(d), respectively.

In Case 2, when initial value $x_{0}=(0.05,0.05,0.05)$, $K_{I}<1.7326, K_{P}=0.5$, and $\tau_{1}>0$, the corresponding oscillation curves of $x_{i}(t)$ are shown in Figure 3(a). System (6) is asymptotically stable at the equilibrium point $E_{0}=$ $(0,0,0)$. We can compute the Hopf bifurcation value $\tau_{10}=$ $0.8322 \mathrm{~s}$. When $K_{I} \geq 1.7326, \tau_{2}=0$, the equilibrium point $E^{*}=\left(x_{1}^{*}, x_{2}^{*}, x_{3}^{*}\right)$ of system (6) is asymptotically stable for $\tau_{1} \in\left[0, \tau_{10}\right)$, and it is unstable when $\tau_{1}>\tau_{10}$ are shown in Figures 4(b) and 4(d), respectively.

In Case 3 , when initial value $x_{0}=(0.05,0.05,0.05), K_{P}=$ 5, $K_{I}=-1.2$, and $\tau_{1}=0$, we obtain the Hopf bifurcation value $\tau_{20}=0.455 \mathrm{~s}$. System (6) is asymptotically stable for $\tau_{1} \in\left[0, \tau_{20}\right)$, and it is unstable when $\tau_{2}$ is more than $\tau_{20}$ at the equilibrium point $E^{*}=\left(x_{1}^{*}, x_{2}^{*}, x_{3}^{*}\right)$, which are shown in Figures 5(b) and 5(d), respectively.

In Case 4 , when initial value $x_{0}=(0.05,0.05,0.05), K_{I}=$ $-1.5326, \tau_{10}=0.8322 \mathrm{~s}$, and $\tau_{2 *}=0.5655 \mathrm{~s}$. The phase portraits are obtained from Theorem 8 in Figures 6(b) and $6(\mathrm{~d})$. When $K_{P}=5$, at Hopf point $E^{*}=\left(x_{1}^{*}, x_{2}^{*}, x_{3}^{*}\right)$ system (6) is stable and for each $\tau_{2}<\tau_{20}$, but close to $\tau_{20}$ there is a stable periodic orbit near the asymptotically stable equilibrium point $E^{*}=\left(x_{1}^{*}, x_{2}^{*}, x_{3}^{*}\right)$. When $\tau_{2}=\tau_{2 *}$ and $\tau_{1}=\tau_{10}$, system (6) has a transversal Hopf point at $E^{*}=$ $\left(x_{1}^{*}, x_{2}^{*}, x_{3}^{*}\right)$ (see Figure 6(d)).

\section{Conclusions}

In the paper, we establish a PI hydroturbine governing system with saturation and double delays. In the case of positive equilibrium point $E^{*}$, the stability of the PI hydroturbine governing system is discussed when the values of the speed control delay and the displacement delay of the servomotor is equal to zero and greater than zero, respectively. The results show that the PI hydroturbine governing system may have unexpected limit cycle oscillation when the delay parameters meet certain conditions. We obtain the scope of three parameters, which determine the stability of periodic solution, the direction of Hopf bifurcation, and the cycle of periodic solutions, respectively. Finally, the theoretical results are validated via the numerical simulation. In addition, a novel approach is proposed to analysis dynamic characteristics of the PI hydroturbine governing system with double delays.

Our work illustrates that the oscillation can be effectively controlled by decreasing speed control delay and setting 


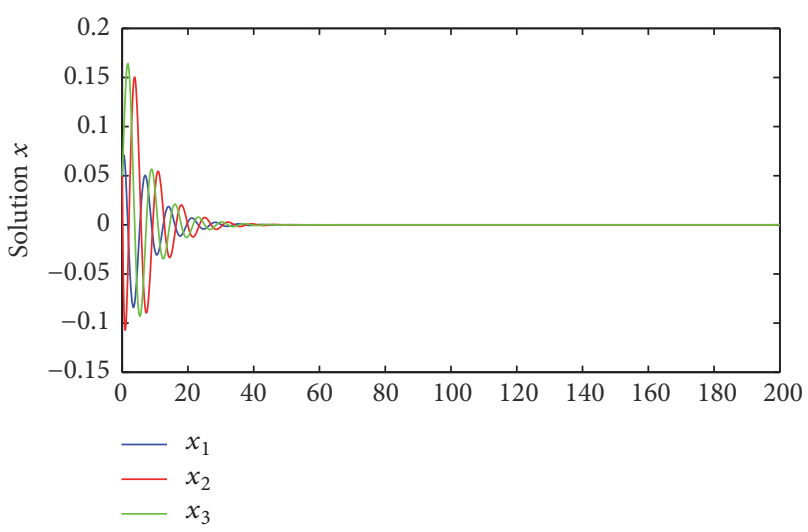

(a)

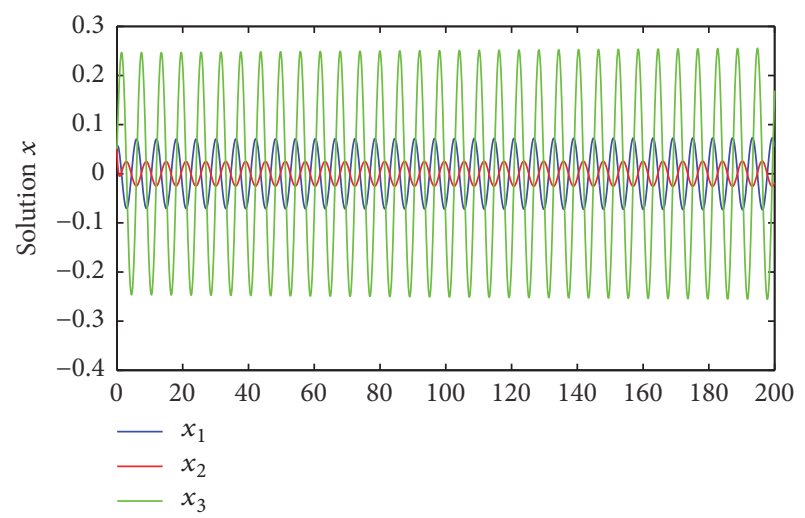

(c)

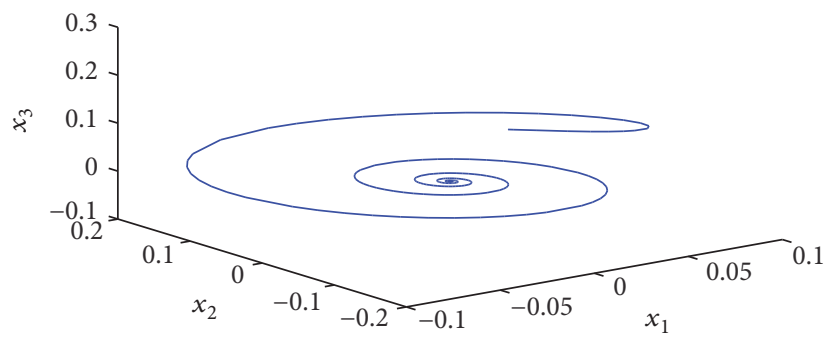

(b)

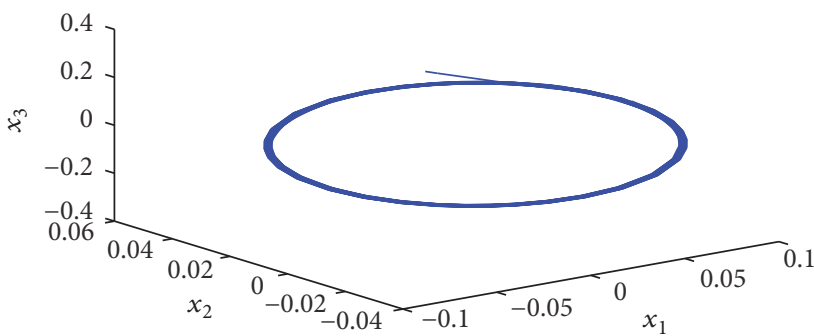

(d)

FIgURE 2: Time waveforms and phase portrait of the PI hydroturbine governing system. (a) Time waveforms with $K_{P}=4.5, K_{I}=-1.326$, and $\tau<0.35 \mathrm{~s}$. (b) The phase portrait with $K_{P}=4.5, K_{I}=-1.326$, and $\tau=0.15 \mathrm{~s}$. (c) Time waveforms of $x_{i}(t)$ with $K_{P}=1.1, K_{I}=-3.6326$, and $\tau \geq 0.35 \mathrm{~s}$. (d) The phase portrait with $K_{P}=1.5$ and $\tau=0.4 \mathrm{~s}$.

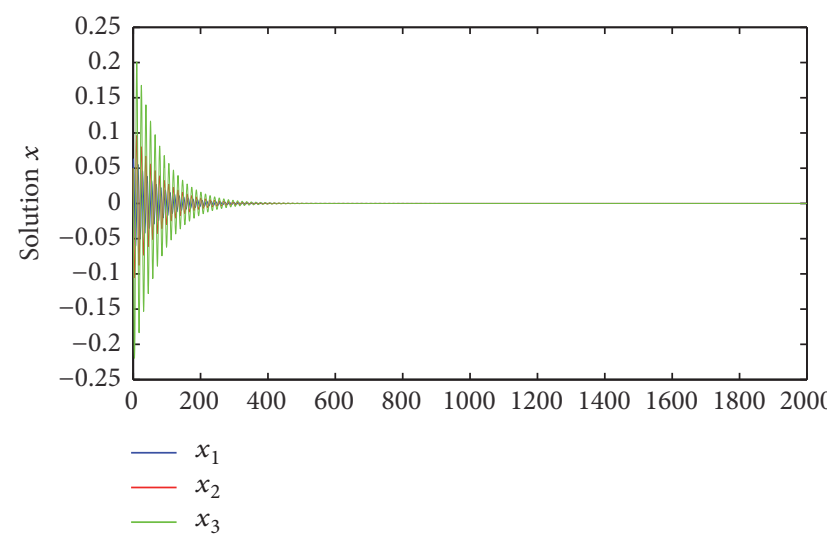

(a)

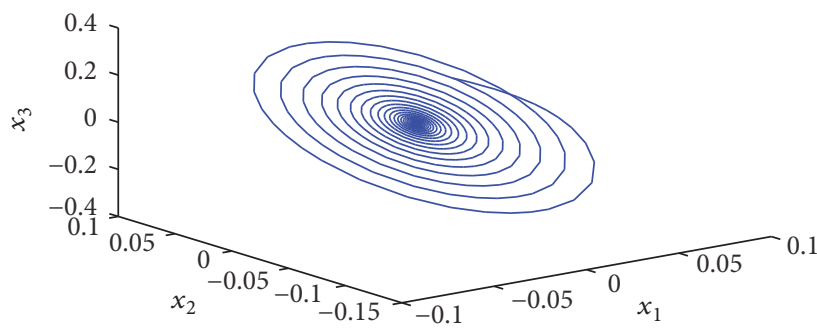

(b)

FIGURE 3: Time waveforms and phase portrait of the PI hydroturbine governing system with the initial value $x_{0}=(0.05,0.05,0.05), K_{I}<$ 1.7326, and $\tau_{2}=0$. (a) Time waveforms of $x_{i}(t)$. (b) The phase portrait with $\tau_{1}>0$.

up the high efficiency of PI controller parameters. A time response device is designed to offset the speed control delay in the hydroturbine governing system. Utilizing data analysis method, the accuracy of servomotor displacement can be improved. The research provides theoretical guidance for hydropower station in maintaining the stability of the hydropower system. In the future work, the model of the PI hydroturbine governing system will be constituted by new materials. The rich nonlinear dynamic characteristics of the system will be analyzed accurately by the theory of fractional 


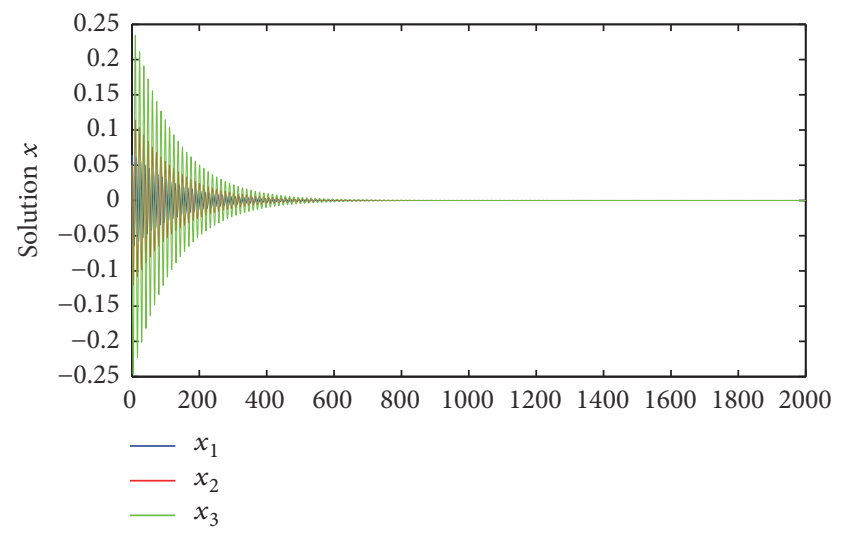

(a)

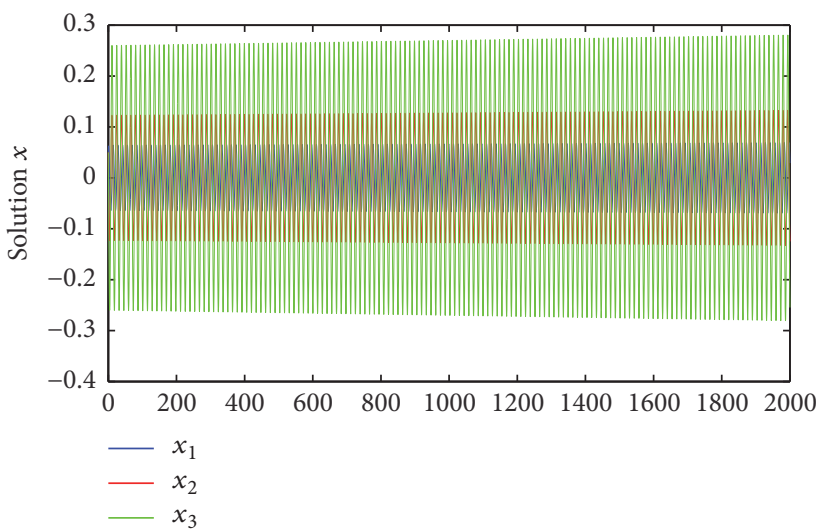

(c)

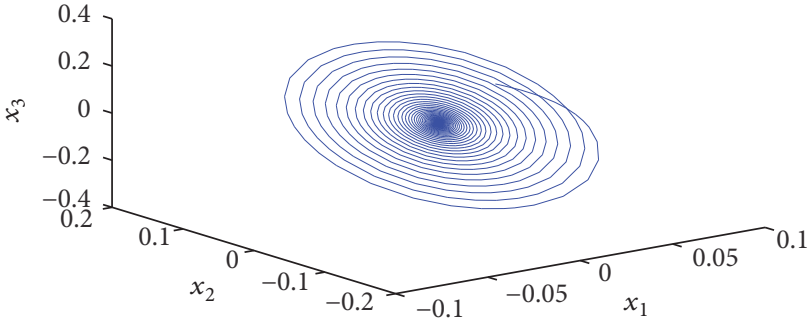

(b)

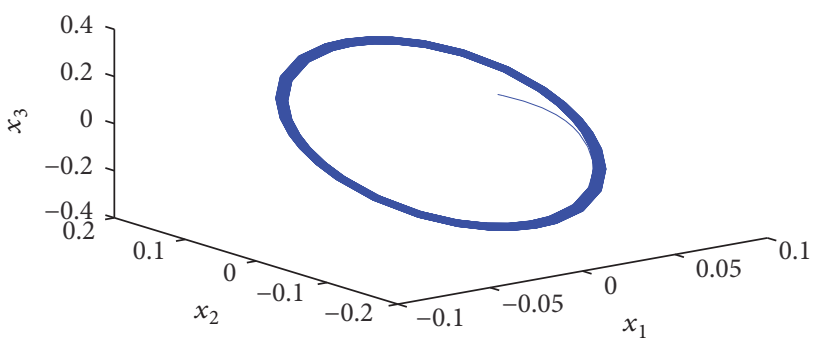

(d)

FIgURE 4: Time waveforms and phase portrait of the PI hydroturbine governing system. (a) Time waveforms with $0<\tau_{1}<\tau_{10}$. (b) The phase portrait with $\tau_{1}=0.75 \mathrm{~s}<\tau_{10}$. (c) Time waveforms for $\tau_{1}=0.92 \mathrm{~s}>\tau_{10}$. (d) The phase portrait with $\tau_{1}=0.84 \mathrm{~s}>\tau_{10}$.

order. These methods and results will provide new ideas to the research of the stability of the hydropower station.

\section{Appendix}

\section{Stability Analysis and Hopf Bifurcation}

According to time delays $\tau_{1}$ and $\tau_{2}$, we analyze the nonlinear dynamic behavior of system (6) under the four different cases.

Case A.1 $\left(\tau=\tau_{1}=\tau_{2} \neq 0\right)$. If $\lambda=i \omega$ is a root of (11), then we have

$$
\begin{aligned}
& \omega A_{5} \sin (\omega \tau)+\left(A_{6}-\omega^{2} A_{4}\right) \cos (\omega \tau)=\omega^{2} A_{1}-A_{3} \\
& \omega A_{5} \cos (\omega \tau)-\left(A_{6}-\omega^{2} A_{4}\right) \sin (\omega \tau)=\omega^{3}-\omega A_{1} .
\end{aligned}
$$

From (A.1), we can obtain

$$
\begin{aligned}
& \sin \omega \tau=\frac{\omega^{5} B_{1}+\omega^{3} B_{2}+\omega B_{3}}{\omega^{4} B_{1}^{2}+\omega^{2} B_{4}+B_{5}} \\
& \cos \omega \tau=\frac{\omega^{4} B_{6}+\omega^{2} B_{7}+B_{8}}{\omega^{4} B_{1}^{2}+\omega^{2} B_{4}+B_{5}},
\end{aligned}
$$

where

$$
\begin{aligned}
& B_{1}=A_{4}, \\
& B_{2}=A_{1} A_{5}-A_{1} A_{4}-A_{6}, \\
& B_{3}=A_{1} A_{6}-A_{3} A_{5}, \\
& B_{4}=A_{5}^{2}-2 A_{4} A_{6}, \\
& B_{5}=A_{6}^{2}, \\
& B_{6}=A_{5}-A_{1} A_{4}, \\
& B_{7}=A_{1} A_{6}-A_{1} A_{5}+A_{3} A_{4}, \\
& B_{8}=-A_{3} A_{6} .
\end{aligned}
$$

Then we have (12). Let $z=\omega^{2}$; we have

$$
z^{5}+z^{4} C_{1}+z^{3} C_{2}+z^{2} C_{3}+z C_{4}+C_{5}=0 .
$$

To analyze the existence and distribution of roots of (A.4), we have (14) and the following:

$$
h^{\prime}(z)=5 z^{4}+4 z^{3} C_{1}+3 z^{2} C_{2}+2 z C_{3}+C_{4} .
$$




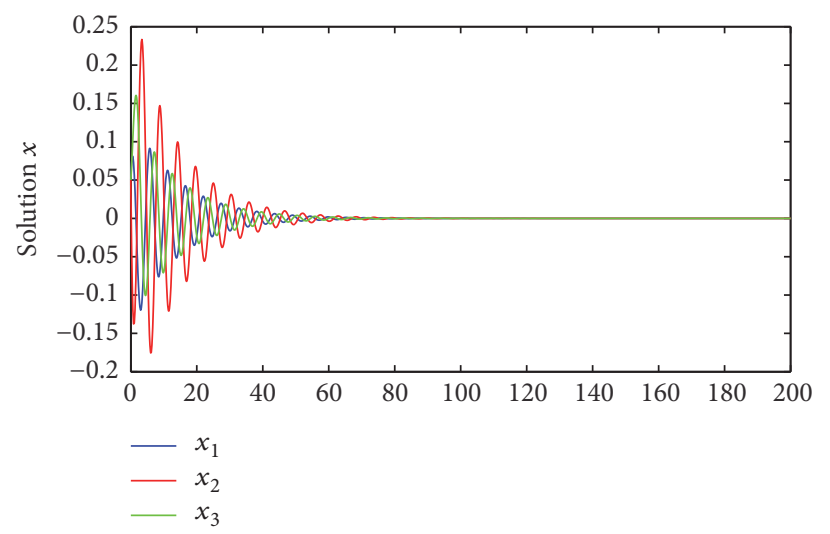

(a)

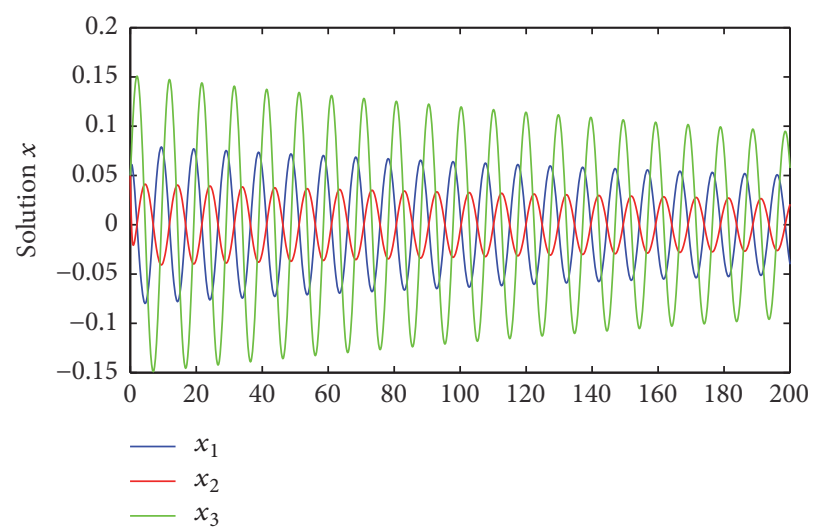

(c)

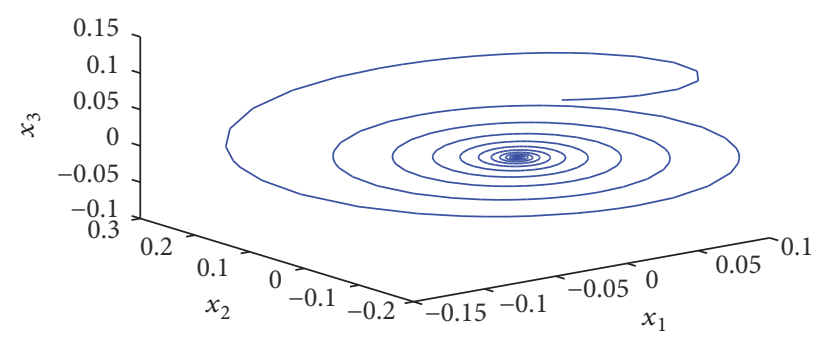

(b)

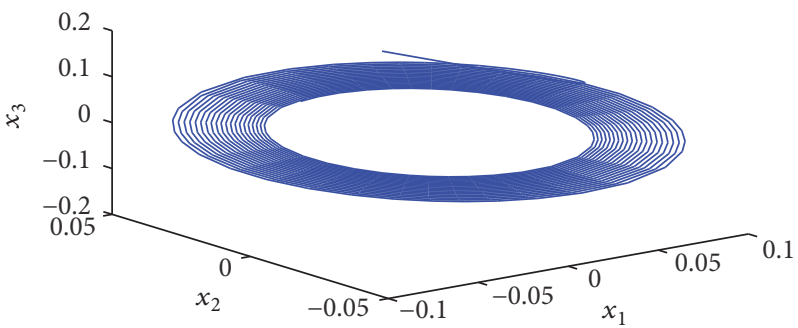

(d)

FIGURE 5: The corresponding oscillation curves and phase portrait of the PI hydroturbine governing system. (a) The corresponding oscillation curves for $K_{P}=5$ and $\tau_{2} \in\left[0, \tau_{20}\right)$. (b) The phase portrait with $K_{P}=5$ and $\tau_{2}=0.35 \mathrm{~s} \in\left[0, \tau_{20}\right)$. (c) The corresponding oscillation curves with $K_{P}=1.5$ and $\tau_{2} \geq \tau_{20}$. (d) The phase portrait with $K_{P}=1.5$ and $\tau_{2}=0.46 \mathrm{~s} \geq \tau_{20}$.

From the above analysis, we have Lemma 2. The proof is as follows.

\section{Proof. We denote}

$$
\begin{aligned}
P= & {\left[3 \lambda^{2}+2 A_{1} \lambda+A_{2}+\left(2 A_{4} \lambda+A_{5}\right) e^{-\lambda \tau}\right]_{\lambda=\omega i} } \\
& =-3 \omega^{2}+A_{2}+A_{5} \cos (\omega \tau)+2 A_{4} \omega \sin (\omega \tau) \\
& +\left(2 A_{4} \omega \cos (\omega \tau)+2 \omega A_{1}-A_{5} \sin (\omega \tau)\right) i:=P_{R} \\
& +P_{I} i \\
Q= & {\left[-\lambda\left(A_{4} \lambda^{2}+A_{5} \lambda+A_{6}\right) e^{-\lambda \tau}\right]_{\lambda=\omega i}=\omega^{3} A_{4} } \\
& \cdot \sin (\omega \tau)+\omega^{2} A_{5} \cos (\omega \tau)-\omega A_{6} \sin (\omega \tau) \\
& +\left(\omega^{3} A_{4} \cos (\omega \tau)-\omega^{2} A_{5} \sin (\omega \tau)\right. \\
& \left.-\omega A_{6} \cos (\omega \tau)\right) i:=Q_{R}+Q_{I} i
\end{aligned}
$$

Taking the derivative of $\lambda$ with respect to $\tau$ in (11), one can get

$$
\begin{aligned}
\left(\frac{d \lambda}{d \tau}\right)^{-1}= & \frac{3 \lambda^{2}+2 A_{1} \lambda+A_{2}+\left(2 A_{4} \lambda+A_{5}\right) e^{-\lambda \tau}}{-\lambda\left(A_{4} \lambda^{2}+A_{5} \lambda+A_{6}\right) e^{-\lambda \tau}} \\
& -\frac{\tau}{\lambda} .
\end{aligned}
$$

By substituting $\lambda=\omega i$ into (A.7) we have

$$
\begin{aligned}
& \left(\frac{d \operatorname{Re} \lambda(\tau)}{d \tau}\right)_{\tau=\tau_{k}^{(i)}}^{-1} \\
& =\operatorname{Re}\left[\frac{3 \lambda^{2}+2 A_{1} \lambda+A_{2}+\left(2 A_{4} \lambda+A_{5}\right) e^{-\lambda \tau}}{-\lambda\left(A_{4} \lambda^{2}+A_{5} \lambda+A_{6}\right) e^{-\lambda \tau}}\right]_{\tau=\tau_{k}^{(i)}} \\
& =\frac{P_{R} Q_{R}+P_{I} Q_{I}}{P_{R}^{2}+Q_{I}^{2}} . \\
& \text { If } H_{2}=P_{R} Q_{R}+P_{I} Q_{I} \neq 0, \text { we can obtain }
\end{aligned}
$$

$$
\left(\frac{d \operatorname{Re} \lambda(\tau)}{d \tau}\right)_{\tau=\tau_{k}^{(i)}} \neq 0
$$

This proves Lemma 2. 


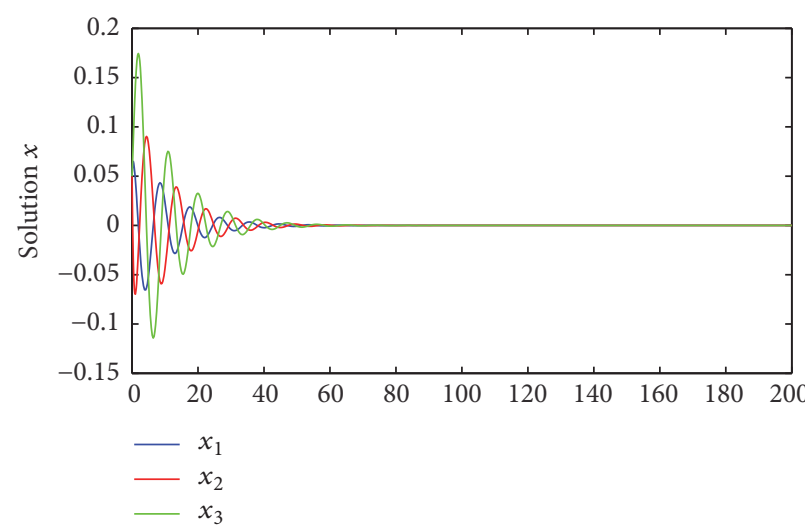

(a)

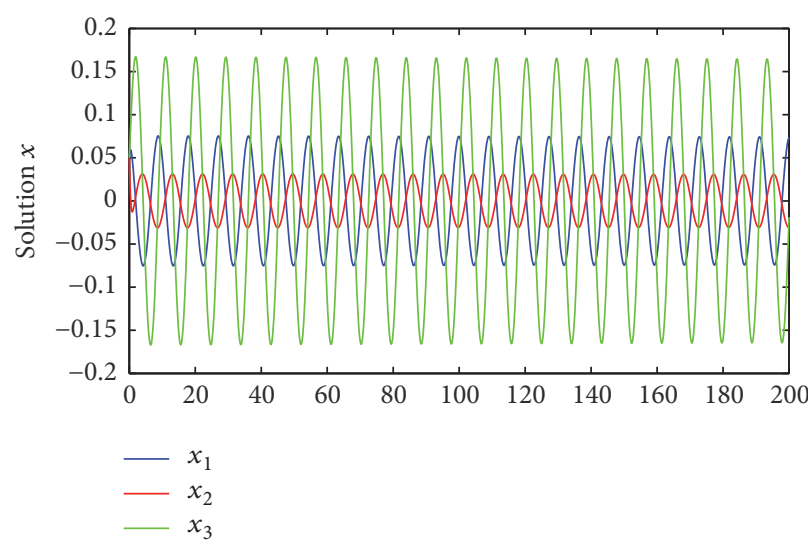

(c)

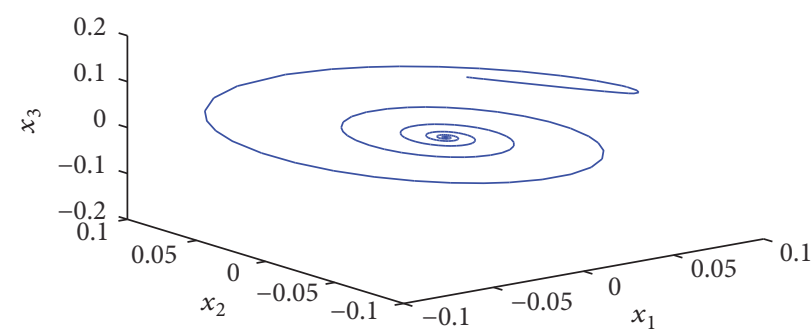

(b)

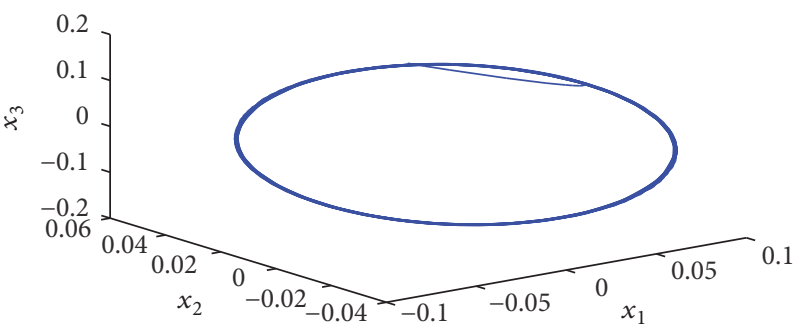

(d)

FIGURE 6: The corresponding oscillation curves and phase portrait of the PI hydroturbine governing system. (a) The corresponding oscillation curves with $K_{P}=5$ and $\tau_{2} \in\left[0, \tau_{20}\right.$ ). (b) The phase portrait with $K_{P}=5$ and $\tau_{2} \in\left[0, \tau_{20}\right)$. (c) The corresponding oscillation curves with $K_{P}=1.2, \tau_{1}=0.01 \mathrm{~s}$, and $\tau_{2} \geq \tau_{2 *}$. (d) Hopf bifurcation occurs for $K_{P}=1.2, \tau_{1}=0.01 \mathrm{~s}$, and $\tau_{2}=\tau_{2 *}$.

Case A.2 $\left(\tau_{1} \neq 0, \tau_{2}=0\right)$. Let $\lambda=i \omega$ be a root of (16), then we have

$$
\begin{aligned}
& A_{3}+A_{6}-\omega^{2} A_{4} \cos (\omega \tau)-\omega^{2} A_{1}+\omega A_{5} \sin (\omega \tau) \\
& \quad+\left(\omega A_{5} \cos (\omega \tau)+\omega A_{2}-\omega^{3}+\omega^{2} A_{4} \sin (\omega \tau)\right) i \\
& =0 .
\end{aligned}
$$

The real and imaginary parts are separated; we obtain

$$
\begin{aligned}
-\omega^{2} A_{4} \cos (\omega \tau)+\omega A_{5} \sin (\omega \tau) & =\omega^{2} A_{1}-A_{3}-A_{6} \\
\omega A_{5} \cos (\omega \tau)+\omega^{2} A_{4} \sin (\omega \tau) & =\omega^{3}-\omega A_{2} .
\end{aligned}
$$

From (A.11), we can obtain

$$
\begin{aligned}
& \sin (\omega \tau) \\
& =\frac{\omega^{4} A_{4}+\omega^{2}\left(A_{1} A_{5}-A_{2} A_{4}\right)-\left(A_{3} A_{5}+A_{5} A_{6}\right)}{\omega^{3} A_{4}^{2}+\omega A_{5}^{2}} \\
& \cos (\omega \tau) \\
& =\frac{\omega^{3}\left(A_{5}-A_{1} A_{4}\right)+\omega\left(A_{3} A_{4}-A_{2} A_{5}+A_{4} A_{6}\right)}{\omega^{3} A_{4}^{2}+\omega A_{5}^{2}} .
\end{aligned}
$$

It follows that

$$
\omega^{8}+\omega^{6} D_{1}+\omega^{4} D_{2}+\omega^{2} D_{3}+D_{4}=0,
$$

where

$$
\begin{aligned}
& D_{1}=\frac{\left(A_{1}^{2} A_{4}^{2}+A_{5}^{2}-A_{4}^{4}-2 A_{2} A_{4}^{2}\right)}{A_{4}^{2}}, \\
& D_{2}=\frac{\left(-2 A_{4}^{2} A_{5}^{2}+A_{1}^{2} A_{5}^{2}+A_{2}^{2} A_{4}^{2}-2 A_{2} A_{5}^{2}-2 A_{1} A_{3} A_{4}^{2}-2 A_{1} A_{4}^{2} A_{6}\right)}{A_{4}^{2}},
\end{aligned}
$$




$$
\begin{aligned}
D_{3} & =\frac{\left(-A_{5}^{4}+A_{3}^{2} A_{4}^{2}+A_{4}^{2} A_{6}^{2}+A_{2}^{2} A_{5}^{2}+2 A_{3} A_{4}^{2} A_{6}-2 A_{1} A_{3} A_{5}^{2}-2 A_{1} A_{5}^{2} A_{6}\right)}{A_{4}^{2}} \\
D_{4} & =\frac{\left(A_{3} A_{5}+A_{5} A_{6}\right)^{2}}{A_{4}^{2}} .
\end{aligned}
$$

Then we obtain (17).

Assume that (17) has positive roots. Without losing generality, we suppose that (17) has four positive roots, which are defined by $z_{1}, z_{2}, z_{3}$, and $z_{4}$. Then, (17) has four positive roots $\omega_{k}=\sqrt{z_{k}}, k=1,2,3,4$.

From the above analysis, we have Lemma 4 . The proof is as follows.

Proof. Taking the derivative of $\lambda$ with respect to $\tau_{1}$ in (16), we have

$$
\begin{aligned}
\left(\frac{d \lambda}{d \tau_{1}}\right)^{-1}= & \frac{\left(3 \lambda^{2}+2 A_{1} \lambda+A_{2}\right) e^{\lambda \tau_{1}}}{\lambda\left(A_{4} \lambda^{2}+A_{5} \lambda\right)} \\
& +\frac{2 A_{4} \lambda+A_{5}}{\lambda\left(A_{4} \lambda^{2}+A_{5} \lambda\right)}-\frac{\tau_{1}}{\lambda}
\end{aligned}
$$

In addition, we have

$$
\begin{aligned}
{\left[\lambda\left(A_{4} \lambda^{2}+A_{5} \lambda\right)\right]_{\lambda=\omega_{k} i} } & =-\omega_{k}^{2} A_{5}-\omega_{k}^{3} A_{4} i \\
{\left[3 \lambda^{2}+2 A_{1} \lambda+A_{2}\right]_{\lambda=\omega_{k} i} } & =A_{2}-3 \omega_{k}^{2}+2 A_{1} \omega_{k} i \\
{\left[2 A_{4} \lambda+A_{5}\right]_{\lambda=\omega_{k} i} } & =A_{5}+2 A_{4} \omega_{k} i .
\end{aligned}
$$

For simplification of analysis, we define $\omega_{k}$ as $\omega$ and $\tau_{k}^{(i)}$ as $\tau_{1}$. From (A.15)-(A.16) and (A.12), we can obtain

$$
\begin{aligned}
& \left(\frac{d \operatorname{Re} \lambda\left(\tau_{10}\right)}{d \tau_{1}}\right)_{\tau_{1}=\tau_{k}^{(i)}}^{-1} \\
& \quad=\operatorname{Re}\left[\frac{\left(3 \lambda^{2}+2 A_{1} \lambda+A_{2}\right) e^{\lambda \tau_{1}}}{\lambda\left(A_{4} \lambda^{2}+A_{5} \lambda\right)}\right]_{\tau_{1}=\tau_{k}^{(i)}}=\frac{1}{\Lambda}\left\{-\omega\left(A_{2}\right.\right. \\
& \left.+\operatorname{Re}\left[\frac{2 A_{4} \lambda+A_{5}}{\lambda\left(A_{4} \lambda^{2}+A_{5} \lambda\right)}\right]_{\tau_{1}=\tau_{k}^{(i)}}=\omega^{2} A_{4} \sin (\omega \tau)\right] \\
& \left.+3 \omega^{2}\right)\left[\omega A_{5} \cos (\omega \tau)+2 A_{1} \omega^{2}\left[\omega A_{5} \sin (\omega \tau)-\omega^{2} A_{4} \cos (\omega \tau)\right]\right. \\
& \left.-2 \omega^{4} A_{4}^{2}-\omega^{2} A_{5}^{2}\right\}=\frac{1}{\Lambda}\left\{4 A_{4}^{2} \omega^{6}+3 \omega^{4}\left(A_{1}^{2} A_{4}^{2}\right.\right.
\end{aligned}
$$

$$
\begin{aligned}
& \left.+A_{5}^{2}-A_{4}^{4}-2 A_{2} A_{4}^{2}\right)+2 \omega^{2}\left(-2 A_{4}^{2} A_{5}^{2}+A_{1}^{2} A_{5}^{2}\right. \\
& \left.+A_{2}^{2} A_{4}^{2}-2 A_{2} A_{5}^{2}-2 A_{1} A_{3} A_{4}^{2}-2 A_{1} A_{4}^{2} A_{6}\right) \\
& +A_{3}^{2} A_{4}^{2}-A_{5}^{4}+A_{4}^{2} A_{6}^{2}+A_{2}^{2} A_{5}^{2}+2 A_{3} A_{4}^{2} A_{6} \\
& \left.-2 A_{1} A_{3} A_{5}^{2}-2 A_{1} A_{5}^{2} A_{6}\right\}=\frac{1}{\Lambda} \cdot h^{\prime}\left(z_{k}\right),
\end{aligned}
$$

where $\Lambda=\omega^{6} A_{4}^{4}+\omega^{4} A_{5}^{2} A_{4}^{2}$. Thus we have

$$
\begin{aligned}
& \operatorname{sign}\left[\frac{d \operatorname{Re} \lambda\left(\tau_{10}\right)}{d \tau_{1}}\right]_{\tau_{1}=\tau_{k}^{(i)}} \\
& \quad=\operatorname{sign}\left[\frac{d \operatorname{Re} \lambda\left(\tau_{10}\right)}{d \tau_{1}}\right]_{\tau_{1}=\tau_{k}^{(i)}}^{-1}=\operatorname{sign}\left[\frac{h^{\prime}\left(z_{k}\right)}{\Lambda}\right] \\
& \quad \neq 0 .
\end{aligned}
$$

Furthermore, since $z_{k}>0$, we conclude that $\left[d \operatorname{Re} \lambda\left(\tau_{10}\right) /\right.$ $\left.d \tau_{1}\right]_{\tau_{1}=\tau_{k}^{(i)}}$ and $h^{\prime}\left(z_{k}\right)$ have the same sign.

Case A.3 $\left(\tau_{1}=0, \tau_{2} \neq 0\right)$. By letting $\lambda=i \omega$ be the root of (21), we have

$$
\begin{aligned}
& \cos \left(\omega \tau_{2}\right)=\frac{\left(A_{1} \omega^{2}-A_{3}\right)}{A_{6}} \\
& \sin \left(\omega \tau_{2}\right)=\frac{\left(A_{2} \omega-\omega^{3}\right)}{A_{6}} .
\end{aligned}
$$

It follows that

$$
\omega^{6}+E_{1} \omega^{4}+E_{2} \omega^{2}+E_{3}=0
$$

where $E_{1}=-2 A_{2}, E_{2}=A_{1}^{2}+A_{2}^{2}-2 A_{1} A_{3}, E_{3}=A_{3}^{2}-A_{6}^{2}$.

If we define $z=\omega^{2}$, we have (22); then we also obtain Lemma 6 and Theorem 7.

Case A.4 $\left(\tau_{1} \neq \tau_{2}, \tau_{1}>0\right.$, and $\left.\tau_{2}>0\right)$. Without losing generality, we consider system (6) in Case 2. Let $\omega i(\omega>0)$ be a root of (8), then we obtain

$$
\begin{gathered}
-\omega^{2} A_{4} \cos \left(\omega \tau_{1}\right)+\omega A_{5} \sin \left(\omega \tau_{1}\right) \\
=\omega^{2} A_{1}-A_{3}-A_{6} \cos \left(\omega \tau_{2}\right) \\
\omega A_{5} \cos \left(\omega \tau_{1}\right)+\omega^{2} A_{4} \sin \left(\omega \tau_{1}\right) \\
=\omega^{3}-A_{2} \omega+A_{6} \sin \left(\omega \tau_{2}\right) .
\end{gathered}
$$

Eliminating $\tau_{2}$, we obtain (24). 
If (24) has finite positive roots, we define the roots of (24) as $\omega_{1}, \omega_{2}, \ldots, \omega_{k}$, such that there is a sequence $\left\{\tau_{2_{i}}^{(j)} \mid j=\right.$ $1,2, \ldots\}$ for every fixed $\omega_{i}(i=1,2, \ldots, k)$.

$$
\tau_{2_{i}}^{(j)}=\frac{1}{\omega_{i}}\left\{\arccos \left(\frac{\omega A_{5} \sin \left(\omega \tau_{1}\right)-\omega^{2} A_{4} \cos \left(\omega \tau_{1}\right)+A_{3}-A_{1} \omega^{2}}{A_{6}}\right)+2 j \pi\right\}, \quad i=1,2, \ldots, k, j=0,1,2, \ldots
$$

then $\pm i \omega_{i}$ is a pair of purely imaginary roots of (24) with $\tau_{2}=$ $\tau_{2_{i}}^{(j)}$.

Let $\tau_{2 *}=\left\{\min \left(\tau_{2_{i}}^{(j)}\right) \mid i=1,2, \ldots, k, j=1,2, \ldots\right\}$, when $\tau_{2}=\tau_{2 *},(24)$ has a pair of purely imaginary roots $\pm i \omega_{*}$ for $\tau_{1} \in\left[0, \tau_{10}\right)$.

Next, in order to verify the transversality condition of Hopf bifurcation, we differentiate (8) with respect to $\tau_{2}$ and substitute $\tau_{2}=\tau_{2 *}$; then we have

$$
\left(\frac{d \operatorname{Re} \lambda\left(\tau_{2}\right)}{d \tau_{2}}\right)_{\tau_{2}=\tau_{2 *}}^{-1}=-\frac{P_{R} Q_{R}+P_{I} Q_{I}}{P_{R}^{2}+Q_{I}^{2}}
$$

where

$$
\begin{aligned}
P_{R}= & A_{6} \cos \left(\omega \tau_{2}\right), \\
P_{I}= & -A_{6} \sin \left(\omega \tau_{2}\right), \\
Q_{R}= & -3 \omega^{2}+\cos \left(\omega \tau_{1}\right)\left(A_{5}+A_{4} \omega^{2}\right) \\
& +\sin \left(\omega \tau_{1}\right)\left(2 A_{2} A_{4} \omega-A_{5} \omega\right), \\
Q_{I}= & 2 A_{1} \omega+\cos \left(\omega \tau_{1}\right)\left(2 A_{2} A_{4}-A_{5} \omega\right) \\
& -\sin \left(\omega \tau_{1}\right)\left(A_{5}+A_{4} \omega^{2}\right), \\
P_{R} Q_{R}+P_{I} Q_{I} \neq & 0 .
\end{aligned}
$$

Therefore, Theorem 8 is true.

\section{Nomenclature}

$G_{t}(s)$ : The transfer function of the guide vane to the hydroturbine moment

$G_{s}(s)$ : The transfer function from the Hydroturbine moment to the speed of the generator

$e_{y}$ : Servomotor stroke transfer coefficient of the turbine torque (p.u.)

$e_{q y}$ : Servomotor stroke transfer coefficient of the flow rate (p.u.)

$e_{q h}$ : The transfer coefficient of the flow rate on the water head of a turbine (p.u.)

$e_{h}$ : The transfer coefficient of the torque on the water head of a turbine (p.u.)

$T_{a}$ : The sum of the machine starting time and load time constant (s)

$K_{P}$ : The proportional component

$\begin{array}{ll}P: & \text { The load perturbation } \\ m_{t}: & \text { Turbine output torque }(\mathrm{N} \cdot \mathrm{m}) \\ C: & \text { The reference input } \\ x_{1}, x_{2}, x_{3}: & \text { The intermediate variables } \\ s: & \text { The strength of the elastic water hammer } \\ & \text { effect } \\ T_{\omega}: & \text { The water inertia time constant of a pres- } \\ e_{x}: & \text { sure guide-water system (s) } \\ y: & \text { The transfer coefficient of a speed on the } \\ e_{g}: & \text { The displacement of the servomotor (m) } \\ \tau_{1}: & \text { The load self-regulation factor (p.u.) } \\ \tau_{2}: & \text { The speed control delay of the generator }(\mathrm{s}) \\ & \text { The displacement-control delay of the ser- } \\ K_{I}: & \text { The integral component } \\ x: & \text { The output of the system } \\ z: & \text { The nonlinear part } \\ N: & \text { The constant of nonlinear part } \\ e, e_{n}: & \text { The intermediate variables. }\end{array}$

Conflicts of Interest

The authors declare that they have no conflicts of interest.

\section{Acknowledgments}

The authors also gratefully acknowledge support from the National Natural Science Foundation (no. 61364001) and Lanzhou Talent Innovation and Entrepreneurship Project (no. 2015-RC-3).

\section{References}

[1] Z. Shen, Analysis of Hydro-Turbine Governing System, Publishing House of Water Conservancy \& Power, Beijing, China, 1991.

[2] H. Huang and Z. Yan, "Present situation and future prospect of hydropower in China," Renewable and Sustainable Energy Reviews, vol. 13, no. 6-7, pp. 1652-1656, 2009.

[3] B. Lehner, G. Czisch, and S. Vassolo, "The impact of global change on the hydropower potential of Europe: a model-based analysis," Energy Policy, vol. 33, no. 7, pp. 839-855, 2005.

[4] S. R. Shakya and R. M. Shrestha, "Transport sector electrification in a hydropower resource rich developing country: energy security, environmental and climate change co-benefits," Energy for Sustainable Development, vol. 15, no. 2, pp. 147-159, 2011.

[5] C. Jiang, Y. Ma, and C. Wang, "PID controller parameters optimization of hydro-turbine governing systems using deterministic-chaotic-mutation evolutionary programming 
(DCMEP)," Energy Conversion and Management, vol. 47, no. 9-10, pp. 1222-1230, 2006.

[6] X. Liu and C. Liu, "Eigenanalysis of oscillatory instability of a hydropower plant including water conduit dynamics," IEEE Transactions on Power Systems, vol. 22, no. 2, pp. 675-681, 2007.

[7] P. Pennacchi, S. Chatterton, and A. Vania, "Modeling of the dynamic response of a Francis turbine," Mechanical Systems and Signal Processing, vol. 29, pp. 107-119, 2012.

[8] J. Li and Q. Chen, "Nonlinear dynamical analysis of hydraulic turbine governing systems with nonelastic water hammer effect," Journal of Applied Mathematics, vol. 2014, Article ID 412578, 11 pages, 2014.

[9] C. K. Sanathanan, "Accurate low order model for hydraulic turbine-penstock," IEEE Transactions on Energy Conversion, vol. 2, no. 2, pp. 196-200, 1987.

[10] D. Chen, C. Ding, X. Ma, P. Yuan, and D. Ba, "Nonlinear dynamical analysis of hydro-turbine governing system with a surge tank," Applied Mathematical Modelling. Simulation and Computation for Engineering and Environmental Systems, vol. 37, no. 14-15, pp. 7611-7623, 2013.

[11] Y. Zeng, Y. Guo, L. Zhang, T. Xu, and H. Dong, "Nonlinear hydro turbine model having a surge tank," Mathematical and Computer Modelling of Dynamical Systems, vol. 19, no. 1, pp. 1228, 2013.

[12] C. S. Li, J. Z. Zhou, J. Xiao, and H. Xiao, "Hydraulic turbine governing system identification using T-S fuzzy model optimized by chaotic gravitational search algorithm," Engineering Applications of Artificial Intelligence, vol. 26, no. 9, pp. 20732082, 2013.

[13] D. J. Ling and Y. Tao, "An analysis of the Hopf bifurcation in a hydroturbine governing system with saturation," IEEE Transactions on Energy Conversion, vol. 21, no. 2, pp. 512-515, 2006.

[14] Z. Galias and M. J. Ogorzalek, "Bifurcation phenomena in second-order digital filter with saturation-type adder overflow characteristic," IEEE Transactions on Circuits and Systems, vol. 37, no. 8, pp. 1068-1070, 1990.

[15] I. Dobson, F. Alvarado, and C. DeMarco, "Sensitivity of Hopf bifurcations to power system parameters," in Proceedings of the 1992 31st IEEE Conference on Decision and Control, pp. 29282933, Tucson, Ariz, USA, December 1992.

[16] A. Nayfeh, A. Harb, and C.-M. Chin, "Bifurcations in a power system model," in Proceedings of the International Symposium on Circuits and Systems (ISCAS '95), vol. 1, pp. 283-286, Seattle, DC, USA, May 1995.

[17] G. J. Silva, A. Datta, and S. P. Bhattacharyya, "PI stabilization of first-order systems with time delay," in PID Controllers for Time-Delay Systems, vol. 37, pp. 2025-2031, Birkhäuser Boston, Boston, Mass, USA, 2005.

[18] H. Shu and Y. Pi, "PID neural networks for time-delay systems," Computers and Chemical Engineering, vol. 24, no. 2-7, pp. 859$862,2000$.

[19] B. Strah, O. Kuljaca, and Z. Vukic, "Speed and active power control of hydro turbine unit," IEEE Transactions on Energy Conversion, vol. 20, no. 2, pp. 424-434, 2005.

[20] C. S. Li and J. Z. Zhou, "Parameters identification of hydraulic turbine governing system using improved gravitational search algorithm," Energy Conversion and Management, vol. 52, no. 1, pp. 374-381, 2011.

[21] A. Khodabakhshian and R. Hooshmand, "A new PID controller design for automatic generation control of hydro power systems," International Journal of Electrical Power and Energy Systems, vol. 32, no. 5, pp. 375-382, 2010.

[22] M. Liu, L. Yang, D. Gan, D. Wang, F. Gao, and Y. Chen, "The stability of AGC systems with commensurate delays," European Transactions on Electrical Power, vol. 17, no. 6, pp. 615-627, 2007.

[23] C.-K. Zhang, L. Jiang, Q. H. Wu, Y. He, and M. Wu, "Delaydependent robust load frequency control for time delay power systems," IEEE Transactions on Power Systems, vol. 28, no. 3, pp. 2192-2201, 2013.

[24] D. Chen, C. Ding, Y. Do, X. Ma, H. Zhao, and Y. Wang, "Nonlinear dynamic analysis for a Francis hydro-turbine governing system and its control," Journal of the Franklin Institute, vol. 351, no. 9, pp. 4596-4618, 2014.

[25] B. Xu, D. Chen, H. Zhang, F. Wang, X. Zhang, and Y. Wu, "Hamiltonian model and dynamic analyses for a hydro-turbine governing system with fractional item and time-lag," Communications in Nonlinear Science and Numerical Simulation, vol. 47, pp. 35-47, 2017.

[26] F. Wang, D. Chen, B. Xu, and H. Zhang, "Nonlinear dynamics of a novel fractional-order Francis hydro-turbine governing system with time delay," Chaos, Solitons and Fractals, vol. 91, pp. 329-338, 2016.

[27] Y. Zhang, J. Cao, and W. Xu, "Stability and Hopf bifurcation of a Goodwin model with four different delays," Neurocomputing, vol. 165, pp. 144-151, 2015.

[28] H. Zhang, D. Y. Chen, B. B. Xu, and F. F. Wang, "Nonlinear modeling and dynamic analysis of hydro-turbine governing system in the process of load rejection transient," Energy Conversion and Management, vol. 90, pp. 128-137, 2015.

[29] W. Ning, C. Jie, and W. Jicheng, "Neuron intelligent control for hydraulic turbine generators," in Proceedings of the 1994 IEEE International Conference on Industrial Technology (ICIT '94), pp. 288-292, Guangzhou, China, December 1994.

[30] W. Group, "Hydraulic turbine and turbine control models for system dynamic studies," IEEE Transactions on Power Systems, vol. 7, no. 1, pp. 167-179, 1992.

[31] S. Čelikovský and G. Chen, "On the generalized Lorenz canonical form," Chaos, Solitons \& Fractals, vol. 26, no. 5, pp. 1271-1276, 2005.

[32] R. Shaw, "Strange attractors, chaotic behavior, and information flow," A Journal of Physical Sciences, vol. 36, no. 1, pp. 80-112, 2014.

[33] J. K. Hale, Theory of Functional Differential Equations, Springer, New York, NY, USA, 1977.

[34] B. D. Hassard, N. D. Kazarinoff, and Y.-H. Wan, Theory and Applications of Hopf Bifurcation, London Mathematical Society Lecture Note Series, Cambridge University Press, Cambridge, UK, 1981. 


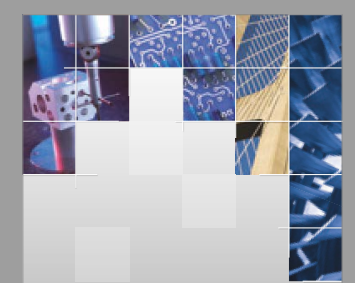

\section{Enfincering}
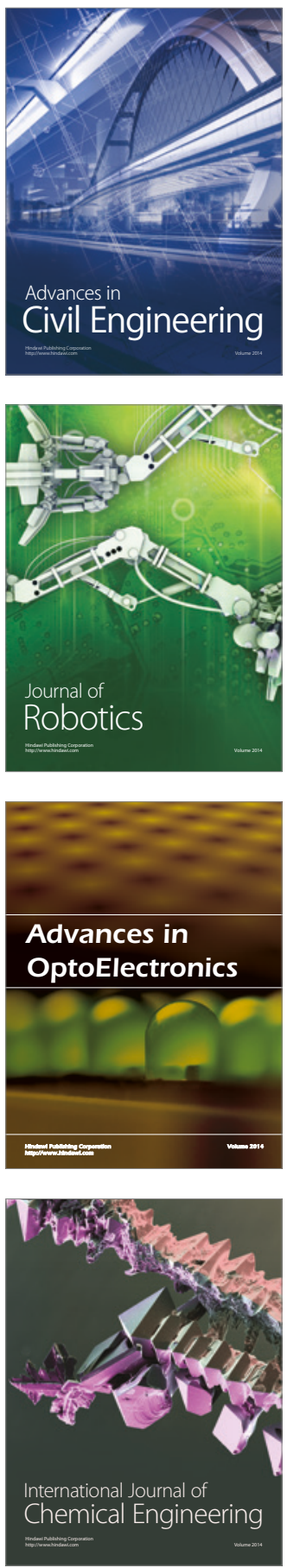

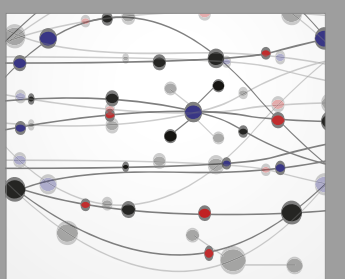

The Scientific World Journal

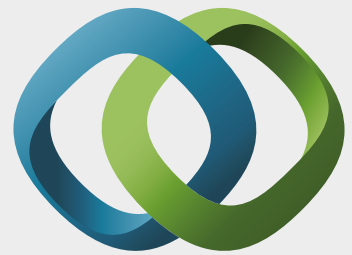

\section{Hindawi}

Submit your manuscripts at

https://www.hindawi.com
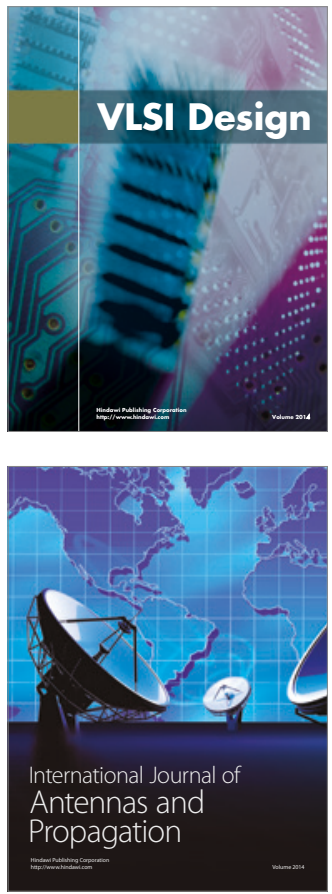

\section{Rotating}

Machinery
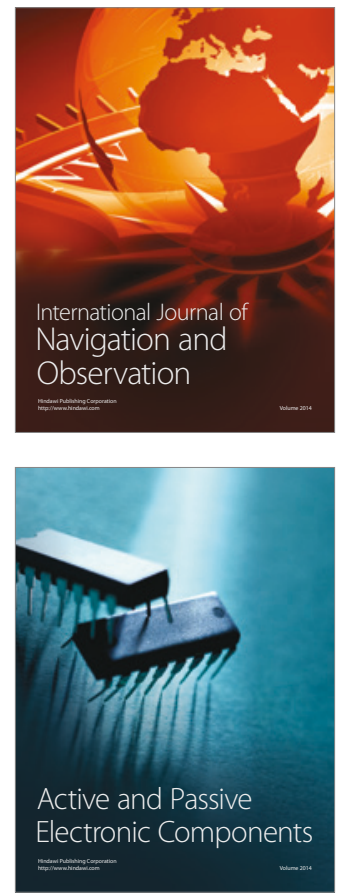
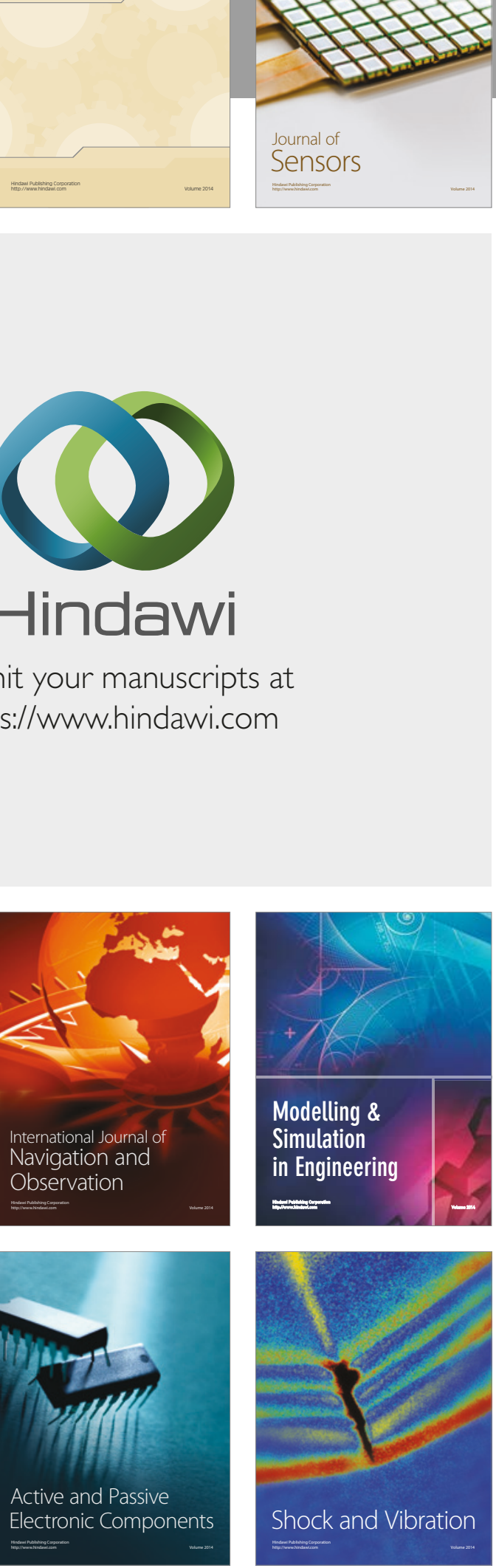
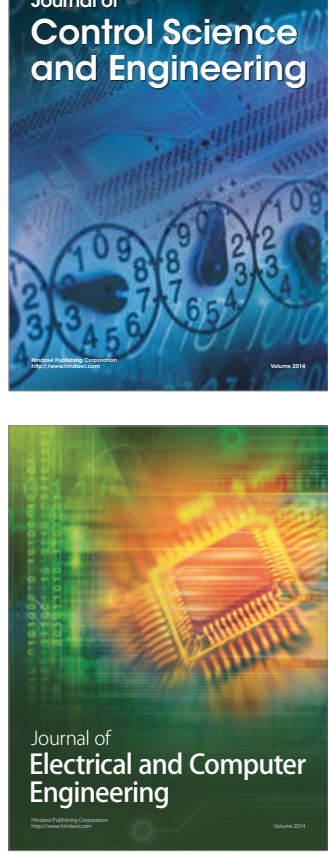

Distributed

Journal of

Control Science

and Engineering
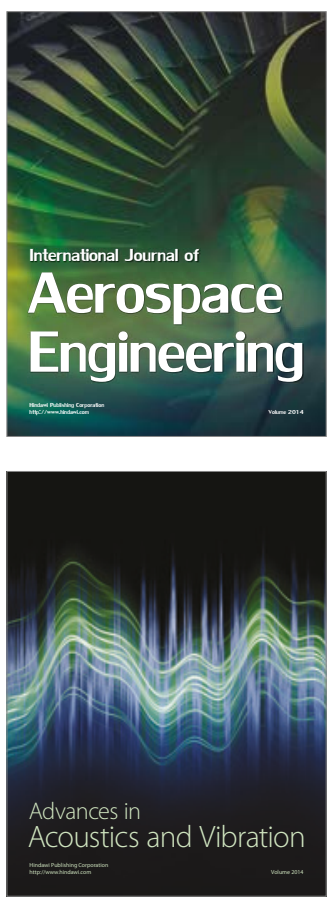

Sensor Networks 